TRANSACTIONS OF THE

AMERICAN MATHEMATICAL SOCIETY

Volume 349, Number 5, May 1997, Pages 1877-1900

S 0002-9947(97)01834-5

\title{
BOUNDARY VALUE MAPS, SZEGÖ MAPS AND INTERTWINING OPERATORS
}

\author{
L. BARCHINI
}

\begin{abstract}
We consider one series of unitarizable representations, the cohomological induced modules $A_{\mathfrak{q}}(\lambda)$ with dominant regular infinitesimal character. The minimal $K$-type $(\tau, V)$ of $A_{\mathfrak{q}}(\lambda)$ determines a homogeneous vector bundle $V_{\tau} \longrightarrow G / K$. The derived functor modules can be realized on the solution space of a first order differential operator $\mathcal{D}_{\Gamma}^{\lambda}$ on $V_{\tau}$. Barchini, Knapp and Zierau gave an explicit integral map $\mathcal{S}$ from the derived functor module, realized in the Langlands classification, into the space of smooth sections of the vector bundle $V_{\tau} \longrightarrow G / K$. In this paper we study the asymptotic behavior of elements in the image of $\mathcal{S}$. We obtain a factorization of the standard intertwining opeartors into the composition of the Szegö map $\mathcal{S}$ and a passage to boundary values.
\end{abstract}

\section{IntRoduction}

Let $G$ be a linear, connected, semisimple Lie group and let $K$ be a maximal compact subgroup of $G$. We assume that $\operatorname{rank} G=\operatorname{rank} K$. In this situation the cohomologically induced representation $A_{\mathfrak{q}}(\lambda)$ with dominant regular infinitesimal character can be realized on solution spaces of invariant differential equations in $G / K$. See ([Wo], [B-2]).

The minimal $K$-type $(\tau, V)$ of $A_{\mathfrak{q}}(\lambda)$ determines an homogeneous vector bundle $V_{\tau} \longrightarrow G / K$. Corresponding to certain choices of positive roots, there is defined a certain homogeneous, first order differential operator $\mathcal{D}_{\uparrow}^{\lambda}$ on $V_{\tau}$. In [BKZ] and [B-1] the authors gave an explicit nonzero integral map $\mathcal{S}$ from the derived functor module, realized in the Langlands classification, to the space of smooth sections of the vector bundle $V_{\tau} \longrightarrow G / K$. The image of $\mathcal{S}$ is annihilated by the differential operator $\mathcal{D}_{1}^{\lambda}$.

When the representation under consideration is in the discrete series of $G$, the differential operator $\mathcal{D}_{\uparrow}^{\lambda}$ coincides with Schmid's differential operator $[\mathrm{S}]$. In this case the $\mathcal{S}$-transform was introduced by Knapp and Wallach in [KW].

The realization of irreducible unitary representations in the $G / K$-picture generalizes Bargmann's formulation of holomorphic discrete series of $S U(1,1)$, [Bar]. In terms of semisimple Lie groups, functions on the circle suggest non-unitary principal series and holomorphic functions on the disk suggest discrete series representations. The $\mathcal{S}$-transform in $[\mathrm{KW}]$ reduces in limiting cases (limits of discrete series for $S U(1,1))$ to the Szegö kernel for the disk. See [K-1]. Thus, we call $\mathcal{S}$ a Szegö map.

Received by the editors February 17, 1995 and, in revised form, October 9, 1995. 1991 Mathematics Subject Classification. Primary 22C05, 22E45, 22E46.

(C)1997 American Mathematical Society 
In this paper we study the asymptotic behavior of functions in the image of the Szegö map and use that information to define a "boundary value map" from the image of $\mathcal{S}$ into an appropriate principal series representation of $G$. We obtain a factorization of the standard intertwining operator into the composition of the Szegö map, as in [BKZ] and [B-1], and a passage to boundary values. Sections 6 and 7 contain the main theorems and definitions of the paper. We use known results on the asymptotic behavior of Eisenstein integrals, and the theory on the constant term in [HC-1]. The interpretation of these results for specific cases that correspond to Szegö maps is new.

The Szegö maps, as in [KW], [Bl-1], [BKZ], [B-1], can be regarded as vector valued versions of the Poisson integrals studied by, among others, Kashiwara et al. in $[\mathrm{KKMOOT}]$. We observe that in [KKMOOT] the composition of the boundary value map and the Poisson transform is a standard intertwining operator.

Brian Blank in [Bl-1], [Bl-2], [Bl-3] has studied Szegö maps and boundary value behavior of limits of discrete series. For groups of real rank one he showed the boundary value map as a factor of the projection operator given by the KnappStein intertwining operator. Blank's treatment of boundary value maps for groups with real rank greater than one uses deep results of Harish-Chandra.

To describe the main results more precisely, we introduce some notation. Let $G$ be a linear connected semisimple Lie group with complexification $G^{\mathbb{C}}$, let $T$ be a torus in $K$, and $L$ be the centralizer of $T$ in $G$. We denote the Lie algebras of the Lie groups $G, K, T, L$ by $\mathfrak{g}_{0}, \mathfrak{k}_{0}, \mathfrak{t}_{0}, \mathfrak{l}_{0}$, etc. and their complexification by $\mathfrak{g}, \mathfrak{k}, \mathfrak{t}, \mathfrak{l}$, etc. We write the Cartan decomposition of $\mathfrak{g}_{0}$ relative to the Cartan involution $\theta$ as $\mathfrak{g}_{0}=\mathfrak{k}_{0} \oplus \mathfrak{p}_{0}$. Our $\theta$-stable parabolic subalgebra of $\mathfrak{g}$ is $\mathfrak{q}=\mathfrak{l} \oplus \mathfrak{u}$ with $\mathfrak{u}$ the unipotent radical.

Extend $\mathfrak{t}_{0}$ to a maximal abelian subspace $\mathfrak{b}_{0}$ of $\mathfrak{k}_{0}$. Let $\lambda$ be in $\mathfrak{b}^{*}$.

We build, as in [BKZ] and [B-1], a maximal abelian subspace $\mathfrak{a}_{0}$ of $\mathfrak{l}_{0} \cap \mathfrak{p}_{0}$ and denote by $\Sigma_{L}$ and $\Sigma_{G}$ the sets of restricted roots for $L$ and $G$. Let $\Sigma_{L}^{+}$and $\Sigma_{G}^{+}$be the sets of positive restricted roots (in an ordering that will be specified later). We let $\rho_{L}$ and $\rho_{G}$ stand for half the sum of the roots in $\Sigma_{L}^{+}$and $\Sigma_{G}^{+}$. In [BKZ] and [B-1] it was shown that $\Sigma_{G}^{+}$can be defined so that $\rho_{L}$ is $\Sigma_{G}^{+}$-dominant.

Define $\mathfrak{n}_{0}$ to be the sum of the root spaces in $\mathfrak{g}_{0}$ for the positive restricted roots, $\overline{\mathfrak{n}}_{0}$ to be $\theta \mathfrak{n}_{0}$, and $N$ and $\bar{N}$ to be the corresponding analytic subgroups. Put $A=\exp \mathfrak{a}_{0}$ and let $P=M A N$ be the canonically associated cuspidal parabolic subgroup of $G$.

Under a positivity condition on $\lambda$, we associate to $A_{\mathfrak{q}}(\lambda)$ a non unitary principal series representation, $\operatorname{ind}_{M A N}^{G}\left(\sigma, \rho_{L}, 1\right)$, where $\sigma$ is a representation in the discrete series of $M$. The Szegö map is an integral operator that carries $\operatorname{ind}_{M A N}^{G}\left(\sigma, \rho_{L}, 1\right)$ continuously in $G$-equivariant fashion into $C^{\infty}\left(G / K, V_{\tau}\right)$.

Let $A^{+}=\left\{a \in A \mid \alpha(\log a)>0\right.$, for every $\left.\alpha \in \Sigma_{G}^{+}\right\}$. We define the boundary value $\mathcal{B}$ of $K$-finite elements $[\mathcal{S} f]$ in the image of the Szegö map by restricting them to $K /(M \cap K) \times A^{+}$and computing the limit

$$
\lim _{\left(a_{+} \rightarrow \infty, P\right)} a_{+}^{\rho_{G}-\rho_{L}} \mathcal{S} f\left(k a_{+}^{-1}\right) .
$$

When $\rho_{L}$ is regular and dominant, then the limit (0.1) can be easily computed by using obsevation (6.4) and Remark 7.2 in this paper combined with standard techniques such as the Dominated Convergence Theorem. The limit provides a "pointwise boundary value" embedding of the Zuckerman modules into the reducible non 
unitary principal series representations, $\operatorname{ind}_{M A N}^{G}\left(\sigma,-\rho_{L}, 1\right)$. When real rank $G=$ real rank $L$, the Boundary Value map is a $G$-equivariant map.

The case of singular $\rho_{L}$ is more delicate. Inspired by the study of the asymptotic expansion near the walls of matrix coefficients of admissible representations, as presented in [K-3], p. 247, we build a parabolic subgroup $P_{1}=M_{1} A_{1} N_{1}$ such that $P \subset P_{1}, P_{1} \cap M=M A_{M_{1}} N_{M_{1}}$ is a cuspidal parabolic subgroup of $M_{1}$ and such that $\left.\rho_{L}\right|_{\mathfrak{a}_{1}}$ is regular and dominant. The idea is first to redo a certain amount of the regular case, replacing objects associated to $P$ by objects associated to $P_{1}$ in order to compute

$$
\lim _{a_{1} \rightarrow \infty, P_{1}} a_{M_{1}}^{\rho_{M_{1}}} a_{1}^{\rho_{1}-\rho_{L}} \mathcal{S} f\left(k a_{M_{1}} a_{1}\right)
$$

for a fixed $a_{M_{1}} \in A_{M_{1}}$. (When the real ranks are different, we must consider the limit (0.2) in the $L^{2}$-sense. See $\S 7$.) The second step is to observe that very well known results on the asymptotic behavior of Eisenstein integrals give information about the asymptotic expansion of (0.2) relative to $P_{1} \cap M$. We define the boundary value of $\mathcal{S} f$ as the leading terms in the asymptotic expansion of $(0.2)$ relative to $P_{1} \cap M$.

Many results concerning the topics touched upon here exist in the literature. Szegö kernels associated to discrete series originated in [K-1] and [KW]. Szegö kernels associated to limits of discrete series were studied in [Bl-1] and [Bl-2]. Szegö kernels associated to $A_{\mathfrak{q}}(\lambda)$ appear in [BKZ], [B-1] and [B-2]. Casselman has obtained embedding results by means of asymptotic coefficients $[\mathrm{C}],[\mathrm{M}]$. Bargmann established the embedding of Limit of discrete series of $S U(1,1)$. Knapp-Okamoto $[\mathrm{K}-\mathrm{O}]$ has constructed for limits of holomorphic discrete series an embedding that is the exact analogue of the Hardy embedding. Among other contributions to the topic are $[\mathrm{GKST}],[\mathrm{Ka}],[\mathrm{Ma}],[\mathrm{Mi}],[\mathrm{Ta}]$.

\section{ACKNOWLEDGMENT}

I am happy to acknowledge Tony Knapp for his advice and assistance. During the last three years he was responding to my numerous e-mail messages, providing answers to both deep and trivial questions, giving advice and recommending references. His help was very important for the completion of this project. I am happy to thank Mike Eastwood for showing me examples of representations realized in the $G / K$-picture where the unitary structure was geometrically understood - in particular, the case of discrete series for $S U(1,1)$ with parameter $n=2$ and the description of the unitary structure by means of an integral defined on the boundary. This example has inspired the work in this paper. I am also grateful to Shif Berhanu for explaining some material in Hörmander's book and for sharing his understanding of some topics in several complex variables.

\section{Preliminaries}

Our underlying group $G$ is assumed to be linear, connected, semisimple, with rank $G=\operatorname{rank} \mathrm{K}$. The equal rank assumption will allow us to refer to the results in [B-2] with no further considerations. We continue with $K, \theta, L, T$, and various Lie algebras as in the introduction.

Let $\Delta=\Delta(\mathfrak{g}, \mathfrak{b})$ be the roots of $\mathfrak{g}$ with respect to $\mathfrak{b}$. We say that a root is compact or noncompact according as its root vector lies in $\mathfrak{k}$ or $\mathfrak{p}$, and we write $\Delta_{c}$ and $\Delta_{n}$ for the set of compact and noncompact roots in $\Delta$. 
Since $\mathfrak{b}_{0}$ centralizes $\mathfrak{t}_{0}, \mathfrak{b}_{0}$ is contained in $\mathfrak{l}_{0}$. Thus, we can speak of sets of roots $\Delta(\mathfrak{u}, \mathfrak{b}), \Delta(\mathfrak{u} \cap \mathfrak{k}, \mathfrak{b}), \Delta(\mathfrak{u} \cap \mathfrak{p}, \mathfrak{b})$ as well as similar sets for $\mathfrak{l}$ and $\overline{\mathfrak{u}}$.

Our choice of a positive system $\Delta^{+}(\mathfrak{g}, \mathfrak{b})$ is so that $\Delta^{+}(\mathfrak{g}, \mathfrak{b})=\Delta^{+}(\mathfrak{l}, \mathfrak{b}) \cup \Delta(\mathfrak{u}, \mathfrak{b})$, with $\Delta^{+}(\mathfrak{l}, \mathfrak{b})$ as in $[\mathrm{B}-1]$. The roots in $\overline{\mathfrak{u}}$ are the negative of the roots in $\mathfrak{u}$. For any of these sets of roots, we let $\delta()$ be half the sum of their members. In particular

$$
\delta(\mathfrak{u} \cap \mathfrak{k})+\delta(\mathfrak{u} \cap \mathfrak{p})=\delta(\mathfrak{u}) .
$$

We choose root vectors $E_{\alpha}$ for the roots in $\Delta(\mathfrak{g}, \mathfrak{b})$, so that the Killing form satisfies

$$
C\left(E_{\alpha}, E_{-\alpha}\right)=1 .
$$

We define $H_{\alpha}$ to be the member in $\mathfrak{b}$ dual to $\alpha$ under the Killing form. Then, by (1.2) it follows that $\left[E_{\alpha}, E_{-\alpha}\right]=H_{\alpha}$.

It is important to observe that in $[\mathrm{BKZ}]$ and $[\mathrm{B}-1]$ the positive system $\Delta^{+}(\mathfrak{l}, \mathfrak{b})$, was given along with a compatible sequence $\alpha_{1}, \alpha_{2} \ldots \alpha_{l}$ of roots in $\Delta_{n}(\mathfrak{l}, \mathfrak{b})$. Thus, our choice of $\Delta^{+}$carries with it a choice of a maximal abelian subspace $\mathfrak{a}_{0}$ of $\mathfrak{p}_{0}$ and a sequence of strongly orthogonal roots $\alpha_{1}, \ldots, \alpha_{l}$ used in defining $\mathfrak{a}_{0}$. In terms of these roots we can define, as in [BKZ], a Cayley transform $\mathbf{c}$ and construct a new $\theta$-stable Cartan subalgebra $\mathfrak{h}_{0}=\mathfrak{g}_{0} \cap \mathbf{c}(\mathfrak{b})$. The subalgebra $\mathfrak{h}_{0}$ is in $\mathfrak{l}_{0}$ since $\mathbf{c}$ is in $\operatorname{Ad} L^{\mathbf{C}}$. (See $[\mathrm{BKZ}]$.) Under the definition $(\mathbf{c} \beta)(H)=\beta\left(\mathbf{c}^{-1} H\right) ; \mathbf{c}$ carries $\Delta(\mathfrak{g}, \mathfrak{b})$ to $\mathbf{c} \Delta=\Delta(\mathfrak{g}, \mathfrak{h})$. For $\beta \in \Delta(\mathfrak{g}, \mathfrak{b})$ we write $X_{\beta}=\mathbf{c}\left(E_{\beta}\right)$. The reader should be warned that $X_{\beta}$ is a root vector for the $\operatorname{root} \mathbf{c} \beta \in \Delta(\mathfrak{g}, \mathfrak{h})$.

Let

$$
\begin{aligned}
\mathfrak{b}_{0}^{\prime} & =\sum_{j=1}^{l} \mathbf{R}\left(i H_{\alpha_{j}}\right) \subset \mathfrak{b}_{0}, \\
\mathfrak{b}_{0}^{-} & =\text {orthocomplement of } \mathfrak{b}_{0}^{\prime \prime} \text { in } \mathfrak{b}_{0} .
\end{aligned}
$$

We denote by $\mathfrak{m}_{0}$ the orthocomplement of $\mathfrak{a}_{0}$ in the centralizer of $\mathfrak{a}_{0}$ in $\mathfrak{g}_{0}$. The subalgebra $\mathfrak{b}_{0}^{-}$is a compact Cartan subalgebra of $\mathfrak{m}_{0}$. If $\Delta^{-}=\left\{\beta \in \Delta(\mathfrak{g}, \mathfrak{b}):\left.\beta\right|_{\mathfrak{b}^{\prime}}=\right.$ $0\}$, then the root system decomposition of $\mathfrak{m}$ is

$$
\mathfrak{m}=\mathfrak{b}^{-} \oplus \sum_{\beta \in \Delta_{-}} \mathbf{C} X_{\beta} .
$$

Each vector $X_{\beta}$ with $\beta \in \Delta_{-}$is either in $\mathfrak{k}$ or $\mathfrak{p}$, and we call $\mathbf{c} \beta$ compact or noncompact accordingly. We define $\Delta^{+}(\mathfrak{m}, \mathfrak{b})=\left\{\mathbf{c} \beta: \beta \in \Delta_{-} \cap \Delta^{+}(\mathfrak{g}, \mathfrak{b})\right\}$.

We choose a positive system $(\mathbf{c} \Delta)^{+}$for $\mathbf{c} \Delta=\Delta(\mathfrak{g}, \mathfrak{b})$ different from the image of $\Delta^{+}$under c. Namely, we list $H_{\alpha_{1}}, \ldots H_{\alpha_{l}}$ as an ordered orthogonal basis of $i \mathfrak{b}_{0}^{\prime}$. We use this basis in lexicographic fashion to determine positivity for members of $\mathbf{c} \Delta$ that do not vanish on $\mathfrak{a}$. (Namely, $\mathbf{c} \beta \in(\mathbf{c} \Delta)^{+}$if $\beta\left(H_{\alpha_{1}}\right)>0$ or $\beta\left(H_{\alpha_{1}}\right)=0$ and $\beta\left(H_{\alpha_{2}}\right)>0$, etc.) For roots supported in $\mathfrak{b}^{-}$we say that $\mathbf{c} \beta \in(\mathbf{c} \Delta)^{+}$if $\beta \in \Delta^{+}(\mathfrak{g}, \mathfrak{b})$. We can consistently define positive restricted roots by saying that $\left.\mathbf{c} \beta\right|_{\mathfrak{a}}>0$ if $\left.\mathbf{c} \beta\right|_{\mathfrak{a}} \neq 0$ and $\mathbf{c} \beta \in(\mathbf{c} \Delta)^{+}$.

We keep the notation in the introduction for various sets of restricted roots and positive restricted roots.

It is crucial to observe that the sequence of roots $\alpha_{1}, \ldots, \alpha_{l}$ can be chosen so that $\rho_{L}$ is dominant for $G$. (See $[\mathrm{BKZ}, \S 12]$ and [B-1].)

We can associate a parabolic subgroup to our Cartan subalgebra $\mathfrak{h}$. Let $\mathfrak{n}_{0}, \overline{\mathfrak{n}}_{0}$, $N, \bar{N}$ and $A$ be as in the introduction. Let $M^{0}$ be the analytic subgroup with Lie algebra $\mathfrak{m}_{0}$. Write $M=Z_{K}(\mathfrak{a}) M^{0}$. Observe that $\mathfrak{b}^{-}$is a maximal abelian subspace 
of $\mathfrak{k} \cap \mathfrak{m}_{0}$ and $B^{-}=\exp \mathfrak{b}^{-}$is a maximal torus in $K \cap M^{0}$. The parabolic subgroup $P=M A N$ is cuspidal.

Let $M^{\#}=M^{0} Z_{M}$ be the product of the identity component and the center of $M$. Lemma 12.30 in [K-3] shows that

$$
M^{\#}=M^{0} F\left(B^{-}\right),
$$

where $F\left(B^{-}\right)$is the finite abelian group in the center of $M$. In particular $F\left(B^{-}\right) \cap$ $M^{0}$ is central in $M^{0}$. Consequently we have

$$
F\left(B^{-}\right) \cap M^{0}=F\left(B^{-}\right) \cap B^{-} .
$$

It is important to note that the parabolic $P=M A N$ has $A$ contained in $L$. Thus, $L \cap M A N=(L \cap M) A(L \cap N)$ is a minimal parabolic subgroup of $L$. Furthermore, it is not difficult to see that $([\mathrm{B}-1,(4.1)])$

$$
F\left(B^{-}\right) \subset L \cap K
$$

Remark 1.1. If $\alpha_{1}, \ldots, \alpha_{l}$ denotes the strongly orthogonal sequence of non-compact roots used in defining $\mathfrak{a}_{0}$, then $\mathbf{c} \alpha_{i} \in \Delta\left(\mathfrak{g}, \mathfrak{a} \oplus \mathfrak{b}^{-}\right)$and $\left.\mathbf{c} \alpha_{i}\right|_{\mathfrak{b}^{-}}=0$. Moreover, $\frac{2\left\langle\mathbf{c} \alpha_{i}, \mathbf{c} \alpha_{i}\right\rangle}{\left|\alpha_{i}\right|^{2}}=2 \neq+1$. Thus, $\left.\mathbf{c} \alpha_{i}\right|_{\mathfrak{a}}$ are useful odd roots in the sense of [K-3, Chap. XIV]. By [K-3, Th. 14:39], $\omega_{0}=s_{\mathbf{c} \alpha_{1}} \ldots s_{\mathbf{c} \alpha_{l}}$ is an element of the Weyl group $W(G: A)$. Moreover, by $\left[K-3\right.$, Th. 14:46] $\omega_{0}$ admits a representative $p$ in $K$. For such a $p, \operatorname{Ad}(p)$ acts as the identity on $\mathfrak{m}_{0}$. Hence, $\omega_{0} \in W(G: A)$ is so that $\omega_{0}\left(\rho_{L}\right)=-\rho_{L}$ and $\omega_{0}$ "acts" as the identity on $\mathfrak{m}_{0}$.

\section{PRINCIPAL SERIES PARAMETERS}

When the dominance condition

$$
\langle\lambda, \alpha\rangle \geqq 0 \text { for all } \alpha \in \Delta^{+}
$$

on $\lambda$ is satisfied, we associate to the Zuckerman module $A_{\mathfrak{q}}(\lambda)$ a principal series representation, according to the Langlands classification. The construction is done in detail in [B-1].

Let $P=M A N$ be the Langlands decomposition of the cuspidal parabolic subgroup introduced in $\S 1$. Put $\Psi=\Delta^{+}(\mathfrak{g}, \mathfrak{h}) \cap \Delta^{-}$, and let $\mathbb{D}(\Psi, \lambda)$ denote the discrete series of $M^{0}$ with Blattner parameter $\lambda+\left.2 \delta(\mathfrak{u} \cap \mathfrak{p})\right|_{\mathfrak{b}^{-}}$and infinitesimal character $\lambda+2 \delta(\mathfrak{u} \cap \mathfrak{p})+\delta_{K}(\Psi)-\delta_{n}(\Psi)$.

Next we define a representation in the discrete series of $M^{\#}=M^{0} F\left(B^{-}\right)$that agrees with $\mathbb{D}(\Psi, \lambda)$ on $M^{0}$. To do so it is enough to define a character $\chi$ on $F\left(B^{-}\right)$ compatible with the action of $\mathbb{D}(\Psi, \lambda)$ on $F\left(B^{-}\right) \cap B^{-}$. Thus, $\mathbb{D}(\Psi, \lambda) \otimes \chi$ is a well defined representation of $M^{\#}$.

Since $\lambda+2 \delta(\mathfrak{u} \cap \mathfrak{p})$ is integral, $\lambda+2 \delta(\mathfrak{u} \cap \mathfrak{p})$ is the differential of a well defined character $\xi_{\lambda+2 \delta(\mathfrak{u} \cap \mathfrak{p})}$ of $B$. We define $\chi$ on $F\left(B^{-}\right)$by means of

$$
\chi(z)=\xi_{\lambda+2 \delta(\mathfrak{u} \cap \mathfrak{p})}\left(u z u^{-1}\right)
$$

where $\mathbf{c}=\operatorname{Ad} u$ is the Cayley transform in $\S 1$.

We take the representation $\sigma$, in the discrete series of $M$, to be

$$
\sigma=\operatorname{ind}_{M \#}^{M}(\mathbb{D}(\Psi, \lambda) \otimes \chi) .
$$

We call $\chi$ the central character of $\sigma$. 
We use the $\mathfrak{a}^{\prime}$-parameter $\rho_{L}$ and the discrete series representation $\sigma$ to form the principal series representation $\operatorname{ind}_{M A N}^{G}\left(\sigma, \rho_{L}, 1\right)$. This is the representation in which $G$ acts by the left regular representation on the space

$$
\left\{f: G \longrightarrow V^{\sigma} \mid f(x m a n)=a^{-\rho_{L}-\rho_{G}} \sigma(m)^{-1} f(x)\right\}
$$

where $V^{\sigma}$ is the space where $\sigma$ acts. $\left(U\left(P, \sigma, \rho_{L}\right)(g) f(x)=f\left(g^{-1} x\right)\right.$.)

We apply double induction formulas to show that our principal series representation is of the form

$$
\operatorname{ind}_{M A A N}^{G}\left([\mathbb{D}(\Psi, \lambda) \otimes \chi], \rho_{L}, 1\right) .
$$

Let $\left(V_{\chi}, \tau_{\chi}\right)$ be an irreducible $K$-representation with highest weight $\chi=\lambda+2 \delta(\mathfrak{u} \cap \mathfrak{p})$ and highest weight vector $\phi$.

Lemma 2.1. The cyclic span of $\phi$ in $V_{\chi}$ under $K \cap M^{\#}, \tilde{V}_{\chi}$, is irreducible under $K \cap M^{\#}$. Namely,

(a) $\phi$ is a highest weight vector under $\mathfrak{m} \cap \mathfrak{k}$, with highest weight $\lambda+\left.2 \delta(\mathfrak{u} \cap \mathfrak{p})\right|_{\mathfrak{b}-}$ and,

(b) $\mathbf{C} \phi$ is a one dimensional space stable under the action of $L \cap K$ (which contains $\left.F\left(B^{-}\right)\right)$.

Proof. See [BKZ], Prop. 3.1.

The discrete series representation $\mathbb{D}(\Psi, \lambda) \otimes \chi$ can be obtained as solution spaces of differential operators acting on $C^{\infty}\left(M^{\#} / K \cap M^{\#}, \tilde{V}_{\chi}\right)$. Indeed, following [KW] we can identify the discrete series representation $\mathbb{D}(\psi, \lambda) \otimes \chi$ with the representation of $M^{\#}$ acting on a subspace $\mathcal{J}_{M}^{\chi}$ of the kernel of Schmid's differential operator $\mathcal{D}_{M \#}$.

Lemma 2.2. If $\omega_{0}$ is as in Remark 1.1, then the representations $\sigma$ and $\omega_{0} \sigma$ are equivalent.

Proof. Let $p$ denote the representative of $\omega_{0}$ in $K$. The discrete series $\omega_{0} \sigma$ is defined as $\omega_{0} \sigma(m)=\sigma\left(p^{-1} m p\right)$. The central character of $\omega_{0} \sigma$ is $\omega_{0} \chi$. (Note that $\omega_{0} \chi$ is well defined since the action of $W(G: A)$ on the center of $M$ is well defined.) In Remark 1.1, we observed that Ad $p$ acts as the identity on $\mathfrak{m}_{0}$. Thus, $\omega_{0} \mathbb{D}(\psi, \lambda)$ is equivalent to $\mathbb{D}(\psi, \lambda)$ as an $M^{0}$-representation. In view of Proposition 4.9 in [K-3], we need to show that $\omega_{0} \chi=\chi$ in order to prove the lemma.

By Lemma 2.1 (b), $\chi$ is the parameter of the one dimensional representation of $F\left(B^{-}\right)$acting on $\mathbf{C} \phi$. The group $F\left(B^{-}\right)$is spanned by the elements $\gamma_{\beta}=\exp \frac{2 i \pi h_{\beta}}{|\beta|^{2}}$ where $\beta$ runs through the restricted roots and where $h_{\beta}$ is the member of $\mathfrak{a}$ dual to $\beta$. For $\gamma_{\beta} \in F\left(B^{-}\right)$we have

$$
\tau\left(\gamma_{\beta}\right) \phi=c \phi
$$

Thus, it is enough to identify the scalar $c$ in equation (2.5). Let $\left\{\alpha_{1}, \ldots, \alpha_{l}\right\}$ be the sequence of strongly orthogonal roots introduced in $\S 1$. Write $h_{\alpha_{j}}=\operatorname{Ad} u_{\alpha_{j}}^{-1} H_{\alpha_{j}}$. We can expand

$$
h_{\beta}=\sum \frac{\left\langle\beta, \mathbf{c} \alpha_{i}\right\rangle}{\left|\mathbf{c} \alpha_{i}\right|^{2}} h_{\alpha_{i}}
$$

then can copy the argument in ([KW], page 344) to show that $c$ is given by $(-1)$ raised to the power $\sum \frac{2\left\langle\lambda+2 \delta(\mathfrak{u} \cap \mathfrak{p}), \alpha_{j}\right\rangle}{\left|\alpha_{j}\right|^{2}} \cdot \frac{\left\langle\beta, \boldsymbol{c} \alpha_{j}\right\rangle}{|\beta|^{2}}$, with the sum extended over those $j$ 
for which $\frac{2\left\langle\beta, \mathbf{c} \alpha_{j}\right\rangle}{\left|\mathbf{c} \alpha_{j}\right|^{2}}$ is even. Thus,

$$
\chi\left(\gamma_{\beta}\right)=\xi_{\lambda+2 \delta(\mathfrak{u} \mathfrak{p})}\left(\gamma_{\beta}\right)=\exp \left(i \pi \sum_{j} \frac{\left\langle\lambda+2 \delta(\mathfrak{u} \cap \mathfrak{p}), \alpha_{j}\right\rangle}{\left|\alpha_{j}\right|^{2}} \cdot \frac{2\left\langle\beta, \mathbf{c} \alpha_{j}\right\rangle}{\left|\alpha_{j}\right|^{2}}\right) .
$$

On the other hand,

$$
\begin{aligned}
\left(s_{\mathbf{c} \alpha_{k}} \chi\right)\left(\gamma_{\beta}\right)= & \exp \left(\sum_{j \neq k} i \pi \frac{\left\langle\lambda+2 \delta(\mathfrak{u} \cap \mathfrak{p}), \alpha_{j}\right\rangle}{\left|\alpha_{j}\right|^{2}} \cdot \frac{2\left\langle\beta, \mathbf{c} \alpha_{j}\right\rangle}{\left|\alpha_{j}\right|^{2}}\right) \\
& \times \exp \left(-i \pi \frac{\left\langle\lambda+2 \delta(\mathfrak{u} \cap \mathfrak{p}), \alpha_{k}\right\rangle}{\left|\alpha_{k}\right|^{2}} \cdot \frac{2\left\langle\beta, \mathbf{c} \alpha_{k}\right\rangle}{\left|\alpha_{k}\right|^{2}}\right) .
\end{aligned}
$$

The sum on the right hand side of (2.7) runs over the $j$ so that $\frac{2\left\langle\beta, \mathbf{c} \alpha_{j}\right\rangle}{\left|\alpha_{j}\right|^{2}}$ is even. By (2.6) and (2.7), we have

$$
\left(s_{\mathbf{c} \alpha_{k}} \chi\right)\left(\gamma_{\beta}\right)=\chi\left(\gamma_{\beta}\right)
$$

\section{KNAPP-STEIN INTERTWINING OPERATORS}

To facilitate exposition and for the convenience of the reader we include in this section some standard results on intertwining operators . References for this section are $[\mathrm{K}-2],[\mathrm{KS}],[\mathrm{KZ}],[\mathrm{K}-3]$ and $[\mathrm{W}-1]$.

Let $\operatorname{ind}_{M A N}^{G}(\pi, \nu, 1)$ be a principal series representation induced from a tempered $M$-representation $\pi$. Let $w \in W(G: A)$ be so that $w \nu=-\bar{\nu},[w \pi]=[\pi]$ and $w^{2}=I$.

Since $w \pi$ is equivalent to $\pi$, it is possible to define $\pi(w)$ as an operator on $V_{\pi}$ in exactly two ways [K-2, page 546]. $\pi(w)$ is a multiple of an operator exhibiting $w \pi$ and $\pi$ as unitarily equivalent.

Lemma 3.1. Let $\nu \in \mathfrak{a}_{0}^{\prime}$ be such that $\operatorname{Re}\langle\nu, \alpha\rangle>0$ for all $\alpha \in \Sigma_{G}^{+}$, and let $\pi$ be a tempered representation of $M$. If $f$ is a $K$-finite function in $\operatorname{ind}_{M A N}^{G}(\pi, \nu, 1)$ and $\psi$ is an $M \cap K$-finite vector in $V_{\pi}$, then

$$
\pi(w) \int_{\bar{N}}\langle f(x p \bar{n}), \psi\rangle d \bar{n}<\infty
$$

for all $x \in G$.

Proof. See $[\mathrm{K}-2, \S 7]$ or $[\mathrm{W}-1,5.3 .1]$.

Remark 3.2. When $P$ is a minimal parabolic subgroup of $G$ and $\nu$ is so that $\operatorname{Re}\langle\nu, \alpha\rangle>0$, for all $\alpha \in \Sigma^{+}$, the integral

$$
\int_{\bar{N}} f(x p \bar{n}) d \bar{n}
$$

converges uniformly for every continuous function in $\operatorname{ind}_{M A N}^{G}(\pi, \nu, 1)$.

We retain the above assumptions on $\pi$ and $\nu$.

Lemma 3.3. If $f$ is a $K$-finite vector in $\operatorname{ind}_{M A N}^{G}(\pi, \nu, 1)$, then there exists a finite dimensional subset $V(f)$ in $V_{M \cap K-\text { finite }}^{\pi}$ such that

$$
\int_{\bar{N}}\langle f(x p \bar{n}), \psi\rangle_{V_{\pi}} d \bar{n}=0
$$

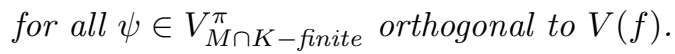


Proof. See $[\mathrm{W}-1,5.3 .2]$.

Lemma 3.4. There exists a (g, K)-intertwining operator $\pi(w) A(w, \pi, \nu)$ from $\operatorname{ind}_{M A N}^{G}(\pi, \nu, 1)$ to $\operatorname{ind}_{M A N}^{G}(\pi,-\bar{\nu}, 1)$ so that for each $K$-finite function $f, k \in K$ and $\psi \in V_{M \cap K-\text { finite }}^{\pi}$

$$
\langle\pi(w) A(w, \pi, \nu) f(k), \psi\rangle_{V^{\pi}}=\int_{\bar{N}}\langle\pi(w) f(k p \bar{n}), \psi\rangle_{V_{\pi}} d \bar{n} .
$$

Proof. See [K-2, §7], [W-1, 5.3.3].

Let $\pi$ be an irreducible tempered representation of $M$. If $P_{1}=M A N_{1}$ and $P_{2}=M A N_{2}$ are two parabolic subgroups of $G$, then we consider

$$
A\left(P_{2}, P_{1}, \pi, \nu\right) f=\int_{\bar{N}_{1} \cap N_{2}} f(k \bar{n}) d \bar{n}
$$

It is possible to show ([W-2, §10], page 13) existence of a constant $c_{\pi}$ such that whenever $\langle\operatorname{Re\nu }, \alpha\rangle \geqq c_{\pi}$ for every $\alpha$ with $\mathfrak{g}_{\alpha} \in \mathfrak{n}_{1} \cap \overline{\mathfrak{n}}_{2}$, then the integral (3.3) converges uniformly.

Lemma 3.5. There exists a function $\eta(P, \bar{P}, \pi, \nu)$, meromorphic in $\nu \in \mathfrak{a}^{\prime}$, such that

$$
A(P, \bar{P}, \pi, \nu) A(\bar{P}, P, \pi, \nu)=\eta(P, \bar{P}, \pi, \nu) I
$$

whenever the left hand side is defined.

Proof. See $[\mathrm{K}-2, \S \mathrm{XIV}]$ and $[\mathrm{W}-2,10.5 .4]$.

Remark 3.6. For a definition of the function $\eta(P, \bar{P}, \pi, \nu)$ see $[\mathrm{K}-2, \S \mathrm{XIV}$, Proposition 14.13] or [W-2, 10.5.7]. If $s \in W(G: A)$ and $k \in K$ is a representative of $s$, then $\eta(P, \bar{P}, k \pi, s \nu)=\eta(P, \bar{P}, \pi, \nu)$. (See [W-2, page 45].)

Following Knapp in $[\mathrm{K}-2, \S 14]$, we define complex valued functions $\gamma(P, \bar{P}, \pi, \nu)$ meromorphic on $\mathfrak{a}^{\prime}$ so that

$$
\gamma(P, \bar{P}, \pi, \nu) \overline{\gamma(\bar{P}, P, \pi, \nu)}=\eta(P, \bar{P}, \pi, \nu) .
$$

Normalized intertwining operators can be defined by means of

$$
\begin{aligned}
\mathcal{A}(P, \bar{P}, \pi, \nu) & =\gamma(P, \bar{P}, \pi, \nu)^{-1} A(P, \bar{P}, \pi, \nu), \\
\mathcal{A}_{P}(w, \pi, \nu) & =\gamma\left(w^{-1} P w, P, \pi, \nu\right)^{-1} A_{P}(w, \pi, \nu) .
\end{aligned}
$$

Lemma 3.7. The operators $\mathcal{A}(P, \bar{P}, \pi, \nu)$ and $\mathcal{A}_{P}(w, \pi, \nu)$ extend meromorphically as functions of $\nu$. They have no pole at $\nu$ when $\operatorname{Re} \nu=0$.

Proof. See [K-2], Prop. 14.20 and Prop. 14.21.

\section{SZEGÖ KERNELS}

The Szegö map $\mathcal{S}$ is an integral operator that carries the representation $\operatorname{ind}_{M M_{A N}}^{G}\left([\mathbb{D}(\Psi, \lambda) \otimes \xi], \rho_{L}, 1\right)$ continuously in $G$-equivariant fashion into the space of smooth $V_{\tau}$-valued sections over $G / K$. Under the identification mentioned after Lemma 2.1 in $\S 2$, the principal series representation is the representation on which 
$G$ acts by the left translation on the space

$$
\begin{aligned}
\mathbf{C}^{\infty}(G, & {\left.[\mathbb{D}(\Psi, \lambda) \otimes \xi], \rho_{L}, 1\right) } \\
\quad & =\left\{f \in \mathbf{C}^{\infty}\left(G, \mathcal{J}_{M^{\#}}^{\chi}\right) \mid \text { with transformation law under } M^{0} F\left(B^{-}\right) A N\right\}
\end{aligned}
$$

with $\mathcal{J}_{M^{\#}}^{\chi} \subset \operatorname{Ker}\left(\mathcal{D}_{M^{\#}}\right)$. Elements of the latter space give rise to elements of $\operatorname{ind}_{K \cap M \# A N}^{G}\left(\tilde{\tau}_{\chi}, \rho_{L}, 1\right)$ by means of the map $g \rightarrow g^{\#}$, where $g^{\#}(x)=g(x)(1)$.

Theorem 4.1. The operator $\mathcal{S}$ given by

$$
(\mathcal{S} f)(x)=\int_{K} \tau_{\chi}(k) f^{\#}(x k) d k
$$

carries $\operatorname{ind}_{M A N}^{G}\left(\sigma, \rho_{L}, 1\right)$ continuously in $G$-equivariant fashion into $\mathbf{C}^{\infty}\left(G / K, V_{\chi}\right)$. The representation of $(\mathfrak{g}, K)$ acting on the space of $K$-finite vectors in the image of $\mathcal{S}$ is infinitesimally equivalent to the Zuckerman module $A_{\mathfrak{q}}(\lambda)$.

Proof. See [BKZ], [B-1], [B-2], [Wo].

Remark 4.2. In [Wo], Wong showed (under a positivity condition on $\lambda$ ) that certain Dolbeault cohomology spaces $H^{s}\left(G / L, \mathbf{C}_{\lambda}^{\#}\right)$ are admissible Fréchet $G$-modules of finite length. Moreover, these cohomology modules are the maximal globalization of the $A_{\mathfrak{q}}(\lambda)$.

In [BKZ] and [B-1], the authors constructed an explicit integral operator $\mathcal{P}$ (a kind of Penrose transform) carrying the space of Dolbeault cohomology in $G$ equivariant fashion into the space of smooth $V_{\tau}$-valued smooth sections on $G / K$. Thus, Image $\mathcal{P}$ admits a natural Fréchet topology and in this topology can be regarded as the maximal globalization of $A_{\mathfrak{q}}(\lambda)$.

In [B-2], the author identifies a special subspace in the image of $\mathcal{P}$. This is the subspace of sections that are in the image of the Szegö map $\mathcal{S}$. The action of $(\mathfrak{g}, K)$ on the space of $K$-finite vectors in the image of $\mathcal{S}$ is infinitesimally equivalent to $A_{\mathfrak{q}}(\lambda)$. In particular, the image of $\mathcal{S}$ is an admissible module of finite length.

The $(\mathfrak{g}, K)$ module $A_{\mathfrak{q}}(\lambda)$ is infinitesimally equivalent to the Langlands quotient of the principal series representation $\operatorname{ind}_{M A N}^{G}\left(\sigma, \rho_{L}, 1\right)$ introduced in $\S 2$. The Langlands classification is usually phrased a little differently, however. Define, as in [VZ],

$$
\begin{aligned}
Z & =\left\{\alpha \text { a root of } \mathfrak{a} \in \mathfrak{g} \mid\left\langle\rho_{L}, \alpha\right\rangle=0\right\}, \\
A_{1} & =\bigcap_{\alpha \in Z} \operatorname{ker} \alpha \subset A, \\
M_{1} A_{1} & =\text { Langlands decomposition of the centralizer of } A \text { in } G, \\
\nu & =\left.\rho_{L}\right|_{\mathfrak{a}_{1}} .
\end{aligned}
$$

We notice that $P \cap M_{1}=M A_{M_{1}} N_{M_{1}}$ is a cuspidal parabolic subgroup of $M_{1}$. (When real rank $G=$ real $\operatorname{rank} L, P \cap M_{1}$ is a minimal parabolic subgroup of $M_{1}$. In general, $P \cap M_{1}$ contains a minimal parabolic subgroup of $M_{1}$.) Define

$$
\pi_{\sigma, \nu}=\operatorname{ind}_{P \cap M_{1}}^{M_{1}}(\sigma, 0,1) .
$$

Since $\left\langle\rho_{L}, \alpha\right\rangle \geq 0$ for all roots $\alpha$ of $\mathfrak{a}$ in $\mathfrak{n}$, there is a unique parabolic subgroup of $G$ having Levi factor $M_{1} A_{1}$ and containing $P$. We write

$$
P \subset P_{1}=M_{1} A_{1} N_{1} \text {. }
$$


By induction in stages,

$$
\operatorname{ind}_{M A N}^{G}\left(\sigma, \rho_{L}, 1\right)=\operatorname{ind}_{M_{1} A_{1} N_{1}}^{G}\left(\operatorname{ind}_{P \cap M_{1}}^{M_{1}}(\sigma, 0,1),\left.\rho_{L}\right|_{\mathfrak{a}_{1}}, 1\right) .
$$

The isomorphism (4.4) is implemented by the map

$$
f \longrightarrow F, \quad F(x)(m)=f(x m) .
$$

By the choice of $P,\left\langle\rho_{L}, \alpha\right\rangle>0$ for all roots $\alpha$ of $\mathfrak{a}_{1}$ in $\mathfrak{n}_{1}$. Since $\pi$ is unitarily induced from a discrete series, it is tempered. It can be shown that $\pi$ is irreducible. Therefore, $\operatorname{ind}_{M_{1} A_{1} N_{1}}^{G}\left(\pi,\left.\rho_{L}\right|_{\mathfrak{a}_{1}}, 1\right)$ is the kind of representation considered in [BW], and it has a unique Langlands quotient, $A_{\mathfrak{q}}(\lambda)$.

We identify the principal series representations in (4.4) with

$$
\operatorname{ind}_{M}^{G}{ }_{A N}\left(\mathbb{D}(\psi, \lambda) \otimes \xi, \rho_{L}, 1\right)=\operatorname{ind}_{M_{1}^{\#} A_{1} N_{1}}^{G}\left(\operatorname{ind}_{M_{1}^{\#} \cap P}^{M_{1}^{\#}}(\mathbb{D}(\psi, \lambda) \otimes \xi, 0,1),\left.\rho_{L}\right|_{\mathfrak{a}_{1}}, 1\right) .
$$

Here $M_{1}^{\#}=M_{1}^{0} F_{1}$, where $F_{1}$ is the finite abelian group generated by elements $\gamma_{\beta}=\exp \left(i \pi|\beta|^{-2} H_{\beta}\right)$ with $\beta$ a root in $\Delta\left(\mathfrak{g}, \mathfrak{a}_{1} \oplus \mathfrak{a}_{M_{1}} \oplus \mathfrak{b}^{-}\right)$vanishing on $\mathfrak{a}_{1} \oplus \mathfrak{b}^{-}$. It follows readily from its definition that $F_{1} \subset F\left(B^{-}\right) \subset L \cap K$. (Compare with [BKZ, §3].)

Lemma 4.3. Let $\left(V_{\chi}, \tau_{\chi}\right)$ be an irreducible $K$-representation with highest weight $\lambda+2 \delta(\mathfrak{u} \cap \mathfrak{p})$ and highest weight vector $\phi$. The cyclic span of $\phi$ in $V_{\chi}$ under $K \cap M_{1}^{\#}$, $\left(\tilde{V}_{\chi}^{1}, \tau_{\chi}^{1}\right)$, is irreducible under $K \cap M_{1}^{\#}$.

Proof . Since $F_{1} \subset L \cap K$, we can copy the proof of Lemma 3.1.

Since the representation $\pi_{\sigma, 0}$ is irreducible, we have

Lemma 4.4. The Szegö map

$$
\tilde{\mathcal{S}}: \operatorname{ind}_{P \cap M_{1}^{\#}}^{M_{1}^{\#}}(\sigma, 0,1) \longrightarrow C^{\infty}\left(M_{1}^{\#} /\left(K \cap M_{1}^{\#}\right), \tilde{V}_{\chi}^{1}\right)
$$

given by

$$
\tilde{\mathcal{S}} g(m)=\int_{K \cap M_{1}^{\#}} \tau_{\chi}^{1}(k)[g(m k)(1)] d k
$$

is an $M_{1}^{\#}$-equivariant isomorphism onto its image.

We observe that $K \cap M^{\#}$ admits an invariant Haar measure, and the integral (4.6) is well defined. We denote by $\mathcal{J}_{M_{1}^{\#}}^{\chi}$ the image of $\tilde{\mathcal{S}}$. Define a $G$-equivariant operator $\mathcal{S}_{1}: \operatorname{ind}_{M_{1} A_{1} N_{1}}^{G}\left(\mathcal{J}_{M_{1}^{\#}}^{\chi},\left.\rho_{L}\right|_{\mathfrak{a}_{1}}, 1\right) \rightarrow C^{\infty}\left(G / K, V_{\chi}\right)$ by means of

$$
\mathcal{S}_{1} H(x)=\int_{K} \tau_{\chi}(k)[H(x k)(1)] d k \quad \text { for } x \in G .
$$

Remark 4.5. If $f \in \operatorname{ind}_{M^{\#} A N}^{G}\left(\mathbb{D}(\psi, \lambda) \otimes \xi, \rho_{L}, 1\right)$, then the function $H$ given by

$$
\begin{aligned}
H(x)(m) & =\int_{K \cap M_{1}^{\#}} \tau_{\chi}^{1}(l)[f(x m l)(1)] d l \\
& =\int_{K \cap M_{1}^{\#}} \tau_{\chi}^{1}(l)[F(x)(m l)(1)] d l
\end{aligned}
$$


for $x \in G$ and $m \in M_{1}^{\#}$, lives in $\operatorname{ind}_{M \# A_{1} N_{1}}^{G}\left(\mathcal{J}_{M_{1}^{\#}}^{\chi},\left.\rho_{L}\right|_{\mathfrak{a}_{1}}, 1\right)$, and

$$
\begin{aligned}
\mathcal{S}_{1} H(x) & =\int_{K} \tau(k) \int_{K \cap M_{1}^{\#}} \tau_{\chi}^{1}(l)[f(x k l)(1)] d l d k \\
& =\int_{K} \int_{K \cap M_{1}^{\#}} \tau(k l)[f(x k l)(1)] d l d k \\
& =\int_{K} \tau(k)[f(x k)(1)] d k \\
& =\mathcal{S} f(x)
\end{aligned}
$$

\section{IRREDUCIBLE TEMPERED REPRESENTATIONS}

In this section we recall several results of Harish-Chandra concerning the harmonic analysis of real reductive groups. The principal references for this section are [HC-1], [HC-2] and $\S 12$ in [W-2]. We have attempted to keep our notation in accordance with that of [W-2] insofar as that was possible. We include a special version of those theorems related to the study of the asymptotic behavior of $K \cap M_{1}$-finite matrix coefficients of the irreducible tempered representation $\pi_{\sigma, i \nu}=\operatorname{ind}_{M_{1} \cap P}^{M_{1}}(\sigma, i \nu, 1)$ with $\nu \in \mathfrak{a}_{0}^{\prime}$.

To each $K \cap M_{1}$-finite vector $w$ in $\operatorname{ind}_{M_{1} \cap P}^{M_{1}}(\sigma, i \nu, 1)$ we associate a $K \cap M_{1}$-finite continuous linear functional on the space of smooth vectors in the principal series by means of

$$
\lambda_{w}\left(\pi_{\sigma, i \nu}(g) v\right)=\int_{K \cap M_{1}}\left\langle\pi_{\sigma, i \nu}(g) v(m), w(m)\right\rangle_{V_{\sigma}} d m .
$$

Here $v$ stands for a smooth vector in the space of realization of the induced representation. Since the representation $\pi_{\sigma, i \nu}$ is tempered, then we have

$$
\left\|\lambda_{w}\left(\pi_{\sigma, i \nu}(g) v\right)\right\| \leqq C_{v, w} \Xi_{M_{1}}(g),
$$

where $\Xi_{M_{1}}$ denotes the Harish-Chandra spherical function for $M_{1}$.

Let $A_{M_{1}}^{+}=\left\{a \in A_{M_{1}} \mid \alpha(\log a)>0\right.$ for all $\Sigma_{M_{1}}^{+}$-dominant roots $\left.\alpha\right\}$, and let $\mathcal{A}_{w}\left(M_{1}\right)$ denote the span of smooth functions on $M_{1}$ satisfying the conditions in [W-2, page 147].

If $a \in A_{M_{1}}^{+}$and $m \in M_{1}$, then we denote by $\Phi_{P \cap M_{1}}\left(a, \lambda_{w}, \pi_{\sigma, i \nu}(m) v\right)$ the leading term in the asymptotic expansion of $\lambda_{w}\left(\pi_{\sigma, i \nu}(a m) v\right)$. (See $[\mathrm{W}-1, \S 4]$ and $[\mathrm{W}-2, \S 12$, page 145].)

\section{Proposition 5.1.}

(1) $\Phi_{P \cap M_{1}} \in \mathcal{A}_{w}\left(M_{1}\right)$.

(2) $\lim _{a_{M_{1}} \rightarrow \infty, P \cap M_{1}}\left\|a_{M_{1}}^{\rho_{M_{1}}} \lambda_{w}\left(\pi_{\sigma, i \nu}\left(a_{M_{1}} m\right) v\right)-\Phi_{P \cap M_{1}}\left(a_{M_{1}}, \lambda_{w}, \pi_{\sigma, i \nu}(m) v\right)\right\|=0$.

(3) $\Phi_{P \cap M_{1}}(., .,$.$) is the unique function in \mathcal{A}_{w}\left(M_{1}\right)$ satisfying (2).

(4) $\Phi_{P \cap M_{1}}\left(a_{M_{1}}, \lambda, \pi_{\sigma, i \nu}(m) v\right)$ is continuous on $\nu$ and $v$ and real analytic in $(a, \nu)$.

Proof. See $[\mathrm{HC}-1, \S 21]$ and $[\mathrm{W}-2,12.3 .5]$. $\left(\lim _{a_{M_{1}} \rightarrow \infty, P \cap M_{1}}\right.$ means that $\log a$ tends to infinity in $\mathfrak{a}_{M_{1}}^{+}$in such a way that every positive root, applied to $\log a$, tends to infinity.) 
The leading term $\Phi_{P \cap M_{1}}\left(a, \lambda, \pi_{\sigma, i \nu}(m) v\right)$ can be related to standard intertwining operators. Indeed, if $W\left(M_{1}: A_{1}\right)=N_{K \cap M_{1}}\left(A_{M_{1}}\right) / Z_{K \cap M_{1}}\left(A_{M_{1}}\right)$, then

$$
\phi_{P \cap M_{1}}\left(a, \lambda, \pi_{\sigma, i \nu}(m) v\right)=\sum_{s \in W\left(M_{1}, A_{M_{1}}\right)} c_{s}(m) a^{i s \nu}
$$

provided $\nu \in \mathfrak{a}_{0}^{\prime}$ is regular.

We set

$$
\Phi_{P \cap M_{1}, s}\left(\lambda, \pi_{\sigma, i \nu}(m) v\right)=c_{s}(m) .
$$

See [W-2], page 159.

Proposition 5.2. If $\nu \in \mathfrak{a}_{0}^{\prime}$ is regular, then $A\left(\bar{P} \cap M_{1}, P \cap M_{1}, \sigma,.\right)$ is holomorphic at $i \nu$ and

$\Phi_{P \cap M_{1}, 1}\left(\lambda_{w}, \pi_{\sigma, i \nu}(m) v\right)=\gamma_{P \cap M_{1}}^{-1}\left\langle A\left(\bar{P} \cap M_{1}, P \cap M_{1}, \sigma, i \nu\right) \pi_{\sigma, i \nu}(m) v(1), w(1)\right\rangle_{V_{\sigma}}$

with $\gamma_{P \cap M_{1}}=\int_{\bar{N}_{1}} a\left(\overline{n_{1}}\right)^{-2 \rho_{M_{1}}} d \overline{n_{1}}$.

Proof. See $[\mathrm{W}-2,12.5 .3]$

Let $s \in W\left(M_{1}, A_{1}\right)$, and let $k \in K \cap M_{1}$ be a representative of $s$.

Proposition 5.3. With the notation just introduced, if $\nu \in \mathfrak{a}_{0}^{\prime}$ is regular, then

$$
\begin{aligned}
& \Phi_{P \cap M_{1}, s}\left(\lambda_{w}, \pi_{\sigma, i \nu}(m) v\right)=\gamma_{P \cap M_{1}}^{-1} \eta^{-1}\left(P \cap M_{1}, k P k^{-1} \cap M_{1}, k \sigma, i s \nu\right) \\
& \times\left\langle A\left(\bar{P} \cap M_{1}, P \cap M_{1}, k \sigma, i s \nu\right) A\left(P \cap M_{1}, k P k^{-1} \cap M_{1}, k \sigma, i s \nu\right) v\left(k^{-1}\right),\right. \\
& \times A\left(k P \cap M_{1} k^{-1}, P \cap M_{1}, k \sigma, i s \nu\right) w\left(k^{-1}\right\rangle_{V_{\sigma}} .
\end{aligned}
$$

Proof. See [W-2, page 163].

Remark 5.4. The functions $\gamma^{-1}\left(\bar{P} \cap M_{1}, P \cap M_{1}, \sigma, i \nu\right) \Phi_{P \cap M_{1}, s}\left(\lambda_{w}, \pi_{\sigma, i \nu}(m) v\right)$ are holomorphic in $\nu \in \mathfrak{a}_{0}^{\prime}$. Moreover,

$$
\lim _{a_{M_{1}} \rightarrow \infty, P \cap M_{1}} a_{M_{1}}^{\rho_{M_{1}}} \gamma^{-1}\left(\bar{P} \cap M_{1}, P \cap M_{1}, \sigma, i \nu\right) \lambda_{w}\left(\pi_{\sigma, 0}\left(m a_{M_{1}}\right) v\right)
$$

exists and is given by

$$
\sum_{s \in W\left(M_{1}, A_{M_{1}}\right)} \phi_{P \cap M_{1}, s}\left(\lambda_{w}, \pi_{\sigma, 0}(m) v\right) \gamma^{-1}\left(\bar{P} \cap M_{1}, P \cap M_{1}, \sigma, 0\right) .
$$

6. Boundary VAlue maps: The CASE REAL RANK $G=$ REAL RANK $L$

In this section we study the asymptotic behavior of elements in the image of the Szegö map and use this information to define a boundary value map. Throughout this section we shall assume that real $\operatorname{rank} G=$ real $\operatorname{rank} L$. This assumption implies that the parabolic subgroup $P=M A N$ introduced in $\S 2$ is minimal. In particular $M$ is compact.

Let $A^{+}=\left\{a \in A \mid \alpha(\log a)>0\right.$ for all $\sum_{G}^{+}$-dominant $\left.\alpha\right\}$. As a motivation we observe that $A^{+}$is an open subset of the set of regular elements $A^{\prime}$ of $G$. $K A^{\prime} K$ is an open dense submanifold of $G$ and the map $(k M, a) \rightarrow k a K$ defines an analytic isomorphism of $K / M \times A^{+}$onto $K A^{\prime} K / K$ which is an open dense submanifold of $G / K$. 
Let $\beta_{1}, \ldots, \beta_{l}$ be the simple roots of $\sum_{G}^{+}$and let $h_{\beta_{1}}, \ldots, h_{\beta_{l}}$ be the dual basis in $\mathfrak{a}$. For any $\mathfrak{a} \in A^{+}$, we define $y_{i}(a)=e^{-\beta_{i}(\log a)}, 1 \leqq i \leqq l$. The map

$$
\begin{aligned}
A^{+} & \longrightarrow(0,1)^{l}, \\
& a \longrightarrow y(a)=\left(y_{1}(a), \ldots, y_{l}(a)\right)
\end{aligned}
$$

is an analytic isomorphism. Let $\Omega=K / M \times(-1,1)^{l}$ and let $\Omega_{+}=K / M \times(0,1)^{l}$. Then, $K / M \times A^{+}$is analytically isomorphic to $\Omega_{+}$. We think of $K / M \times A^{+}$as a cone or "wedge" in $\Omega$ with "edge" $K / M \times\{0\}$. We will refer to $K / M$ as the "Martin boundary" of $G / K$. In view of this observation it is natural to define the boundary value of $\mathcal{S} f$ in the image of $\mathcal{S}$ by means of

$$
\lim _{a_{+} \rightarrow \infty, P} a_{+}^{\rho_{G}-\rho_{L}} \mathcal{S} f\left(k a_{+}\right)
$$

provided the limit exists. Here $\lim _{a_{+} \rightarrow \infty, P}$ means $\log (a)$ tends to infinity in $\mathfrak{a}^{+}$in such a way that every positive root, applied to $\log (a)$, tends to infinity.

When $\rho_{L}$ is regular dominant, the domain of the Szegö map consists of smooth vectors in the induced representation $\operatorname{ind}_{M A N}^{G}\left(\sigma, \rho_{L}, 1\right)$.

If $w_{0}=\pi s_{\mathbf{c} \alpha_{i}} \in W(G: A)$ and $p \in K$ is a representative, then the intertwining map

$$
\begin{aligned}
T\left(w_{0}\right): V_{\sigma} & \longrightarrow V_{\sigma}, \\
v & \longrightarrow P_{\sigma}\left[\tau\left(w_{0}\right)^{-1} v\right],
\end{aligned}
$$

where $P_{\sigma}$ denotes the projection from $V_{\tau}$ to $V_{\sigma}$, exhibits the representations $\sigma$ and $\omega_{0} \sigma$ of $M$ as unitarily equivalent. We define

$$
\sigma\left(w_{0}\right)=T\left(w_{0}\right) .
$$

Elements in the image of $\mathcal{S}$ are smooth sections of the vector bundle $V_{\tau} \rightarrow G / K$. Thus, $P_{\sigma}\left[\mathcal{S} f\left(k a_{+} p\right)\right]=P_{\sigma}\left[\tau\left(p^{-1}\right) \mathcal{S} f\left(k a_{+}\right)\right]=\sigma\left(w_{0}\right) \mathcal{S} f\left(k a_{+}\right)$. On the other hand, $w_{0}^{2}=1$ and $w_{0}$ acts by $-I$ on $\mathfrak{a}$. Hence,

$$
P_{\sigma}\left[\mathcal{S} f\left(k a_{+}\right)\right]=\sigma\left(w_{0}\right) \mathcal{S} f\left(k p a_{+}^{-1}\right) .
$$

It is formula (6.4) together with standard results that allow us to prove

Lemma 6.1. If $\rho_{L}$ is in the open restricted positive Weyl chamber, then

$$
\lim _{a_{+} \rightarrow \infty, P} a_{+}^{\rho_{G}-\rho_{L}} P_{\sigma}\left[\mathcal{S} f\left(k a_{+}\right)\right]=\sigma\left(w_{0}\right) A\left(w_{0}, \rho_{L}, \sigma\right) f(k) .
$$

The limit (6.5) converges pointwise and uniformly for $k \in K$.

When $\rho_{L}$ is singular, the analysis in Lemma 6.1 runs into difficulties. To avoid such difficulties we build a parabolic subgroup $P_{1}=M_{1} A_{1} N_{1}$ with $P \subset P_{1}$ as in $\S 4$. If $a_{M_{1}} \in A_{M_{1}}^{+}$and $a_{1} \in A_{1}^{+}$, then $a_{M_{1}} a_{1} \in A^{+}$. The idea is first to fix $a_{M_{1}} \in A_{M_{1}}^{+}$and to redo a certain amount of Lemma 6.1 , replacing objects associated to $M A N$ by objects associated to $P_{1}=M_{1} A_{1} N_{1}$. Indeed, by Remark 4.5, to $f \in \operatorname{ind}_{M A N}^{G}\left(\sigma, \rho_{L}, 1\right)$ we can associate a function $H \in \operatorname{ind}_{M_{1}^{\#} A_{1} N_{1}}^{G}\left(\mathcal{J}_{M_{1}}^{\chi},\left.\rho\right|_{\mathfrak{a}_{1}}, 1\right)$ so that

$$
\mathcal{S} f(x)=\mathcal{S}_{1} H(x)=\int_{K} \tau(k)[H(x k)(1)] d k
$$


and

$$
\begin{aligned}
P_{\sigma}\left[\mathcal{S} f\left(k a_{+}\right)\right] & =\sigma\left(w_{0}\right) \mathcal{S} f\left(k a_{M_{1}} p a_{1}^{-1}\right) \\
& =\sigma\left(w_{0}\right) \int_{K} \tau(l)\left[H\left(k a_{M_{1}} p a_{1}^{-1} l\right)(1)\right] d l .
\end{aligned}
$$

We refer to Remark 4.5 for the definition of the function $H$.

We have three decompositions, $G=K M_{1} A_{1} N_{1}, G=K A N$ and $M_{1}=$ $\left(M_{1} \cap K\right) A_{M_{1}} N_{M_{1}}$. We let $H_{1}, H, H_{M_{1}}$ be the respective $H$-functions and $\rho_{1}, \rho_{G}, \rho_{M_{1}}$ be the respective $\rho$-functions. Some relations among these are

$$
\begin{gathered}
\rho_{G}=\rho_{1}+\rho_{M_{1}}, \\
H=H_{1}+H_{M_{1}}, \\
\rho_{G}-\rho_{L}\left(H_{1}(x)\right)=\rho_{1}-\rho_{L}\left(H_{1}(x)\right), \\
\rho_{G}-\rho_{L}\left(H_{M_{1}}\right)=\rho_{1}-\rho_{L}\left(H_{1}(x)\right)+\rho_{M_{1}}\left(H_{M_{1}}(x)\right), \quad \text { since } \rho_{1}-\rho_{L}\left(H_{M_{1}}(x)\right)=0 .
\end{gathered}
$$

For each $x \in G$, we write $x=k(x) \mu_{1}(x) a_{1}(x) n_{1}(x)$ with $\left(k, \mu_{1}, a_{1}, n_{1}\right) \in K \times$ $\exp \left(\mathfrak{m}_{1} \cap \mathfrak{p}\right) \times A_{1} \times N_{1}$.

An argument similar to the one in the proof of Lemma 6.1 gives us

Lemma 6.2. With the notation just introduced,

$$
\lim _{a_{1} \rightarrow \infty, P_{1}} a_{1}^{\rho_{1}-\rho_{L}} \mathcal{S}_{1} H\left(k a_{M_{1}} p a_{1}^{-1}\right)=\left[A\left(\bar{P}_{1}, P_{1}, \pi_{\sigma, 0},\left.\rho_{L}\right|_{\mathfrak{a}_{1}}\right) H\right]\left(k a_{M_{1}} p\right)(1) .
$$

The limit converges pointwise for $k \in K$ and $a_{M_{1}} \in A_{M_{1}}^{+}$.

In order to define the boundary value of an element in the image of the Szegö map, in the $\rho_{L}$ singular case, we shall introduce deformations $\mathcal{S}_{\epsilon}$ and $\mathcal{S}_{1, \epsilon}$ of our Szegö maps. Following the work of others, for example the work of Kashiwara et al. in [KKMOOT], we shall define the bounday value of $\mathcal{S} f$ to be the leading terms in the asymptotic expansion of

$$
\lim _{a_{1} \rightarrow \infty, P_{1}} a_{M_{1}}^{\rho_{M_{1}}} a_{1}^{\rho_{1}-\rho_{L}} \sigma\left(\omega_{0}\right) \mathcal{S}_{\epsilon} f_{\epsilon}\left(k a_{M_{1}} p a_{1}\right)
$$

relative to $P \cap M_{1}$, as $\epsilon \rightarrow 0$.

The results summarized in $\S 5$ will give us the desired leading term.

Proof of Lemma 6.1. We have

$$
\mathcal{S} f\left(k p a_{+}^{-1}\right)=\int_{K} \tau_{\lambda+2 \delta(\mathfrak{u} \cap \mathfrak{p})}(l) f\left(k p a_{+}^{-1} l\right) d l .
$$

We make the change of variables that passes from $K$ to $\bar{N} \times M$, and observe that $f\left(k p a_{+}^{-1} \bar{n}\right)=e^{-\rho_{L}-\rho_{G} H(\bar{n})} f\left(k p a_{+}^{-1} \kappa(\bar{n})\right)$, to obtain

$$
\begin{aligned}
\mathcal{S} f\left(k a_{+}^{-1}\right) & =a_{+}^{\rho_{G}+\rho_{L}} \int_{\bar{N}} e^{-\rho_{G}+\rho_{L} H(\bar{n})} \tau\left(\kappa(\bar{n}) f\left(k p a_{+}^{-1} \bar{n} a_{+}\right) d \bar{n}\right. \\
=a_{+}^{\rho_{G}+\rho_{L}} \operatorname{det}\left(\left.\operatorname{Ad}\left(a_{+}^{-1}\right)\right|_{\bar{n}}\right)^{-1} \int_{\bar{N}} e^{-\rho_{G}+\rho_{L} H\left(a_{+} \bar{n} a_{+}^{-1}\right)} e^{-\rho_{G}-\rho_{L} H(\bar{n})} & \times \tau\left(\kappa\left(a_{+} \bar{n} a_{+}^{-1}\right)\right) f(k p \kappa(\bar{n})) d \bar{n} .
\end{aligned}
$$

On the other hand, $\operatorname{det}\left(\operatorname{Ad}\left(\left.a_{+}^{-1}\right|_{\bar{n}}\right)^{-1}=a_{+}^{-2 \rho_{G}}\right.$. Thus,

$$
\mathcal{S} f\left(k p a_{+}^{-1}\right)=a_{+}^{-\rho_{G}+\rho_{L}} \int_{\bar{N}} e^{-\rho_{G}+\rho_{L} H\left(\bar{n}^{a_{+}}\right)} e^{-\rho_{L}-\rho_{G} H(\bar{n})} \tau\left(\kappa\left(\bar{n}^{a_{+}}\right)\right) f(k p \kappa(\bar{n})) d \bar{n}
$$


where $\bar{n}^{a_{+}}=a_{+} \bar{n} a_{+}^{-1}$ and $d \bar{n}$ is normalized so that $\int_{\bar{N}} e^{-2 \rho_{G} H(\bar{n})} d \bar{n}=1$. By $(6.6)$, we have

$$
\begin{aligned}
& \lim _{a_{+} \rightarrow \infty, P} a_{+}^{\rho_{G}-\rho_{L}} P_{\sigma}\left[\mathcal{S} f\left(k a_{+}\right)\right] \\
& \quad=\lim _{a_{+} \rightarrow \infty, P} \sigma\left(w_{0}\right) \int_{\bar{N}} e^{-\rho_{G}+\rho_{L} H\left(\bar{n}^{a_{+}}\right)} e^{-\rho_{G}-\rho_{L} H(\bar{n})} \tau\left(\kappa\left(\bar{n}^{a_{+}}\right)\right) f(k p \kappa(\bar{n})) d \bar{n} .
\end{aligned}
$$

We use the dominated convergence theorem to show that the limit and integral in (6.7) can be interchanged. In fact, since $f$ is a continuous function on $K$ the integrand is (6.7) is dominated above by

$$
\begin{aligned}
\| e^{-\rho_{G}-\rho_{L} H(\bar{n})} e^{-\rho_{G}+\rho_{L} H\left(\bar{n}^{a_{+}}\right)} & \tau\left(\kappa\left(\bar{n}^{a_{+}}\right)\right) f(k p \kappa(\bar{n})) \| \\
& \leqq C e^{-\rho_{L}-\rho_{G} H(\bar{n})} e^{-\rho_{G}+\rho_{L} H\left(\bar{n}^{a}+\right)} .
\end{aligned}
$$

On the other hand, $\bar{n}^{a_{+}} \in \bar{N}$, and the real parameter $\rho_{G}-\rho_{L}$ is $\Sigma_{G}^{+}$-dominant. Thus, the right hand side of $(6.8)$ is bounded above by $e^{\left(-\rho_{L}-\rho_{G}\right)(H(\bar{n}))}$.

Since we have assumed that $\rho_{L}$ is in the open positive restricted Weyl chamber and since our the parabolic subgroup $P=M A N$ is minimal, the function $e^{-\rho_{G}-\rho_{L} H(\bar{n})}$ is integrable. (See for example [K-3, Lemma 7.23].)

It is immediate to check that for each fixed $x \in G$, the pointwise limit

$$
\lim _{\left(a_{+} \rightarrow \infty, P\right)} a_{+}^{\rho_{G}-\rho_{L}} \mathcal{S} f\left(x a_{+}\right)
$$

exists, and it is given by $\sigma\left(w_{0}\right) A\left(w_{0}, \rho_{L}, \sigma\right) f(x)$. Thus, we can reinterpret the boundary value map as a $G$-equivariant embedding of the image of $\mathcal{S}$ into the principal series representation $\operatorname{ind}_{M A N}^{G}\left(\sigma,-\rho_{L}, 1\right)$. In other words, the diagram of $G$-equivariant maps

$$
\operatorname{ind}_{M A N}^{G}\left(\sigma, \rho_{L}, 1\right) \stackrel{\sigma\left(w_{0}\right) A\left(w_{0}, \sigma, \rho_{L}\right)}{\longrightarrow} \operatorname{ind}_{M A N}^{G}\left(\sigma, \rho_{L}, 1\right)
$$

commutes. Since Image $\mathcal{S}$ is irreducible, then $\mathcal{B}$ is an injective map.

Proof of Lemma 6.2. We make the change of variables that passes from $K$ to $\bar{N}_{1} \times$ $\left(M_{1} \cap K\right)$, and observe that

$$
H\left(k a_{M_{1}} p a_{1}^{-1} \bar{n}_{1}\right)(1)=e^{-\rho_{L}-\rho_{1}\left(H_{1}\left(\bar{n}_{1}\right)\right)} H\left(k a_{M_{1}} p a_{1}^{-1} \kappa\left(\bar{n}_{1}\right)\right)\left(\mu_{1}\left(\bar{n}_{1}\right)\right),
$$

in order to show that

$$
\begin{aligned}
& \mathcal{S}_{1} H\left(k a_{M_{1}} p a_{1}^{-1}\right) \\
& \quad=a_{1}^{\rho_{1}+\rho_{L}} \int_{\bar{N}_{1}} e^{-\rho_{1}+\rho_{L} H_{1}\left(\bar{n}_{1}\right)} \tau\left(\kappa\left(\bar{n}_{1}\right)\right)\left\{H\left(k a_{M_{1}} p a_{1}^{-1} \bar{n}_{1} a_{1}\right)\left(\mu_{1}\left(\bar{n}_{1}\right)^{-1}\right)\right\} d \bar{n}_{1} .
\end{aligned}
$$

By a change of variables we have

$$
\begin{aligned}
& \lim _{a_{1} \rightarrow \infty, P_{1}} a_{1}^{-\rho_{1}-\rho_{L}} \mathcal{S}_{1} H\left(k a_{M_{1}} p a_{1}^{-1}\right) \\
&=\lim _{a_{1} \rightarrow \infty, P_{1}} \int_{\bar{N}_{1}} e^{-\rho_{1}+\rho_{L} H_{1}\left(\bar{n}_{1}^{a_{1}}\right)} e^{-\rho_{1}-\rho_{L} H_{1}\left(\bar{n}_{1}\right)} \\
& \quad \times \tau\left(\kappa\left(\bar{n}_{1}^{a_{1}}\right)\right)\left\{H\left(k a_{M_{1}} p \kappa\left(\bar{n}_{1}\right)\right)\left(\mu_{1}\left(\bar{n}_{1}\right) \mu_{1}\left(\bar{n}_{1}^{a_{1}}\right)^{-1}\right)\right\} d \bar{n}_{1}
\end{aligned}
$$


where $\bar{n}_{1}{ }^{a_{1}}=a_{1} \bar{n}_{1} a_{1}^{-1}$. The limit (6.9) is computed by means of standard techniques. For example see Wallach's exposition on a result of Langlands in [W-1, 5.3.4]. For the benefit of the non-experts, we briefly indicate how to proceed.

By Remark 4.5 in $\S 4$,

$$
\begin{aligned}
& H\left(k a_{M_{1}} p \kappa\left(\bar{n}_{1}\right)\right)\left(\mu_{1}\left(\bar{n}_{1}\right) \mu_{1}\left(\bar{n}_{1}^{a_{1}}\right)^{-1}\right) \\
& =\int_{K \cap M_{1}^{\#}} \tau_{\chi}^{1}(m)\left\{F\left(k a_{M_{1}} p \kappa\left(\bar{n}_{1}\right)\right)\left(\mu_{1}\left(\bar{n}_{1}\right) \mu_{1}\left(\bar{n}_{1}^{a_{1}}\right)^{-1} m\right)\right\} d m_{1}
\end{aligned}
$$

where $F$ is a function in $\operatorname{ind}_{M_{1}^{\#} A_{1} N_{1}}^{G}\left(\operatorname{ind}_{P \cap M_{1}^{\#}}^{M_{1}^{\#}}(\sigma, 0,1),\left.\rho_{L}\right|_{a_{1}}, 1\right)$. Moreover, if $\left\{\phi_{i}\right\}$ is an orthonormal basis for $V_{\sigma}$, then

$$
\begin{aligned}
& H\left(k a_{M_{1}} p \kappa\left(\bar{n}_{1}\right)\right)\left(\mu_{1}\left(\bar{n}_{1}\right) \mu_{1}\left(\bar{n}_{1}^{a_{1}}\right)^{-1}\right) \\
& \quad=\sum_{i} \int_{K \cap M_{1}^{\#}}\left\langle F\left(k a_{M_{1}} p \kappa\left(\bar{n}_{1}\right)\left(\mu_{1}\left(\bar{n}_{1}\right) \mu_{1}\left(\bar{n}_{1}^{a_{1}}\right)^{-1} m\right), \tau_{\chi}^{1}\left(m^{-1}\right) \phi_{i}\right\rangle d m_{1} \quad \phi_{i} .\right.
\end{aligned}
$$

Define $\psi_{i}(m)=\tau_{\chi}^{1}\left(m^{-1}\right) \phi_{i}$ for $m \in M_{1}^{\#} \cap K$. The functions $\psi_{i}$ give rise to $M_{1}^{\#} \cap K$ finite vectors in $\pi_{\sigma, 0}$. Thus, we can rewrite (6.10) as

$$
\begin{aligned}
H\left(k a_{M_{1}} p \kappa\left(\bar{n}_{1}\right)\right)\left(\mu_{1}\left(\bar{n}_{1}\right)\right. & \left.\mu_{1}\left(\bar{n}_{1}^{a_{1}}\right)^{-1}\right) \\
& =\sum_{i} \overline{\left\langle\pi_{\sigma, 0}\left(\mu_{1}\left(\bar{n}_{1}\right) \mu_{1}\left(\bar{n}_{1}^{a_{1}}\right)^{-1}\right) \psi_{i}, F\left(k a_{M_{1}} p \kappa\left(\bar{n}_{1}\right)\right)(.)\right\rangle} \phi_{i} .
\end{aligned}
$$

The representation $\pi_{\sigma, 0}$ is tempered, so by (5.1) we conclude that

$$
\| H\left(k a_{M_{1}} p \kappa\left(\operatorname{barn}_{1}\right)\left(\mu_{1}\left(\bar{n}_{1}\right) \mu_{1}\left(\bar{n}_{1}^{a_{1}}\right)^{-1}\right) \| \leqq C \Xi_{M_{1}}\left(\mu_{1}\left(\bar{n}_{1}\right) \mu_{1}\left(\bar{n}_{1}^{a_{1}}\right)^{-1}\right) .\right.
$$

Thus, the integral on the right hand side of (6.9) is bounded above by

$$
\int_{\bar{N}_{1}} e^{-\rho_{1}+\rho_{L} H_{1}\left(\bar{n}_{1}^{a_{1}}\right)} e^{-\rho_{1}+\rho_{L} H_{1}\left(\bar{n}_{1}\right)} \Xi_{M_{1}}\left(\mu_{1}\left(\bar{n}_{1}\right) \mu_{1}\left(\bar{n}_{1}^{a_{1}}\right)^{-1}\right) d \bar{n}_{1} .
$$

Since $\left.\rho_{L}\right|_{\mathfrak{a}_{1}}$ is in the positive restricted Weyl chamber, the integral (6.12) converges uniformly for $a_{1} \in A_{1}^{+}$. See [HC-1, page 49], [L, Lemma 3.12]. Hence, we can interchange limit and integration in (6.9).

Corollary 6.3. Let $f$ be a vector in $\operatorname{ind}_{M}^{G}{ }_{A N}\left(\sigma, \rho_{L}, 1\right)$ and let $F$ be the corresponding function in $\operatorname{ind}_{M_{1}^{\#} A_{1} N_{1}}^{G}\left(\operatorname{ind}_{M_{1}^{\#} \cap P}^{M_{M}^{\#}}(\sigma, 0,1),\left.\rho_{L}\right|_{\mathfrak{a}_{1}}, 1\right)$. If $P=M A N$ is a minimal parabolic subgroup of $G$, then for each $a_{M_{1}} \in A_{M_{1}}$, we have

$$
\begin{aligned}
\lim _{a_{1} \rightarrow \infty, P_{1}} & a_{M_{1}}^{\rho_{M_{1}}} a_{1}^{\rho_{1}-\rho_{L}} \mathcal{S} f\left(k a_{M_{1}} a_{1}\right) \\
& =\sum_{i}\left\{\int_{\bar{N}_{1}} a_{M_{1}}^{\rho_{M_{1}}}\left\langle\pi_{\sigma, 0}\left(a_{M_{1}}\right) F\left(k p \bar{n}_{1}\right)(.), \psi_{i}(.)\right\rangle_{L^{2}\left(K \cap M_{1}^{\#}\right)} d \bar{n}_{1}\right\} \tau\left(p^{-1}\right) \phi_{i} .
\end{aligned}
$$

With the notation of sections 5 and 6 , we have

Proposition 6.4. For each $\bar{n}_{1} \in \bar{N}_{1}$, let $\Phi_{P \cap M_{1}^{\#}}\left(a_{M_{1}}, \psi_{i}, F\left(k p \bar{n}_{1}\right)().\right)$ be the leading term in the analytic expansion of $\left\langle\pi_{\sigma, 0}\left(a_{M_{1}}\right) F\left(k p \bar{n}_{1}\right)(.), \psi_{i}(.)\right\rangle_{L^{2}\left(K \cap M_{1}^{\#}\right)}$. Then, 
when real rank $G=$ real rank $L$, we have

$$
\begin{aligned}
\lim _{a_{M_{1}} \rightarrow \infty, P \cap M_{1}^{\#}} \| & \lim _{a_{1} \rightarrow \infty, P_{1}} a_{M_{1}}^{\rho_{M_{1}}} a_{1}^{\rho_{1}-\rho_{L}} \mathcal{S} f\left(k a_{M_{1}} a_{1}\right) \\
& \quad-\sum_{i}\left\{\int_{\bar{N}_{1}} \Phi_{P \cap M_{1}^{\#}}\left(a_{M_{1}}, \psi_{i}, F\left(k p \bar{n}_{1}\right)(.)\right) d \bar{n}_{1}\right\} \tau\left(p^{-1}\right) \phi_{i} \|=0 .
\end{aligned}
$$

Proof. The convergence of the integrals in (6.13) follows from well known results

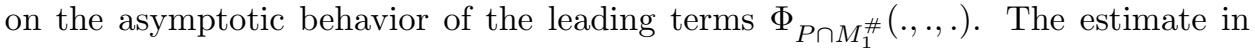
[W-2, Lemma 12.3.2 (1)], followed by an argument similar to the one in [W-1, 4.5.6] shows that such integrals are bounded above by

$$
\int_{\bar{N}_{1}} e^{-\rho_{1}(1+\epsilon) H_{1}\left(\bar{n}_{1}\right)} \Xi_{M_{1}}\left(\mu_{1}\left(\bar{n}_{1}\right)\right) d \bar{n}_{1},
$$

which converges by [HC-1, Corollary of Lemma 32.2]. By Lemma 6.2 and linearity of $\Phi_{P \cap M_{1}^{\#}}\left(a_{M_{1}}, \psi_{i}, v\right)$ on $V$, it follows that $(6.13)$ is bounded above by

$$
\begin{aligned}
\lim _{a_{M_{1}} \rightarrow \infty, P \cap M_{1}^{\#}} & \sum_{i} \int_{\bar{N}_{1}} e^{-\rho_{L}-\rho_{1} H_{1}\left(\bar{n}_{1}\right)} \\
\left.\times \| a_{M_{1}}^{\rho_{M_{1}}}\left\langle\pi_{\sigma, 0}\left(a_{M_{1}} \mu_{1}\left(\bar{n}_{1}\right)^{-1}\right)\right) F\left(k p \kappa\left(\bar{n}_{1}\right)\right)(.), \psi_{i}(.)\right\rangle_{L^{2}\left(K \cap M_{1}^{\#}\right)} & \quad-\Phi_{P \cap M_{1}^{\#}}\left(a_{M_{1}}, \psi_{i}, \pi_{\sigma, 0}\left(\mu_{1}\left(\bar{n}_{1}\right)^{-1}\right) F\left(k p \kappa\left(\bar{n}_{1}\right)\right) \| d \bar{n}_{1} .\right.
\end{aligned}
$$

By [W-2, Lemma 12.3.2 (3)], the integrand in (6.14) is dominated by

$$
e^{-\rho_{L}-\rho_{1} H_{1}\left(\bar{n}_{1}\right)}\left(1+\left\|\log \mu_{1}\left(\bar{n}_{1}\right)^{-1}\right\|\right)^{d} \Xi_{M_{1}}\left(\mu_{1}\left(\bar{n}_{1}\right)\right) .
$$

We proceed as in the first part of the proof to show that (6.15) belongs to $L^{1}\left(\bar{N}_{1}\right)$. Thus, we can interchange limit and integration. Now the proposition follows from Prop. 5.1 (2).

Let $\epsilon>0$, and consider the principal series representation

$$
\operatorname{ind}_{M \# A N}^{G}\left(\sigma, \rho_{L}+\epsilon \rho_{M_{1}}, 1\right) .
$$

For $f_{\epsilon} \in \operatorname{ind}_{M}^{G}{ }_{A N}\left(\sigma, \rho_{L}+\epsilon \rho_{M_{1}}, 1\right)$ let

$$
F_{\epsilon} \in \operatorname{ind}_{M_{1}^{\#} A_{1} N_{1}}^{G}\left(\operatorname{ind}_{M_{1}^{\#} \cap P}^{M_{1}^{\#}}\left(\sigma, \epsilon \rho_{M_{1}}, 1\right),\left.\rho_{L}\right|_{\mathfrak{a}_{1}}, 1\right) \text { be } F_{\epsilon}(x)(m)=f_{\epsilon}(x m) .
$$

Define $H_{\epsilon}$ by

$$
\begin{aligned}
H_{\epsilon}(x)(m) & =\int_{K \cap M_{1}^{\#}} \tau_{\chi}^{1}\left(m_{1}\right) F_{\epsilon}(x)\left(m m_{1}\right) d m_{1} \\
& =\sum_{i}\left\langle\pi_{\sigma, i \epsilon \rho_{M_{1}}}\left(m^{-1}\right) F_{\epsilon}(x)(.), \psi_{i}(.)\right\rangle_{L^{2}\left(K \cap M_{1}^{\#}\right)} \phi_{i} .
\end{aligned}
$$

Define

$$
\begin{aligned}
\mathcal{S}_{\epsilon} f_{\epsilon}(x) & =\int_{K} \tau_{\chi}(k) f_{\epsilon}(x k) d k \\
\mathcal{S}_{1, \epsilon} H_{\epsilon}(x)(m) & =\int_{K} \tau_{\chi}(k) H_{\epsilon}(x k)(m) d k .
\end{aligned}
$$

We can show as in Remark 4.5 that $\mathcal{S}_{\epsilon} f_{\epsilon}(x)=\mathcal{S}_{1, \epsilon} H_{\epsilon}(x)(1)$. $\mathcal{S}_{\epsilon}$ is a deformation of the Szegö map $\mathcal{S}$ in that $\mathcal{S}_{0}=\mathcal{S}$. 
Definition 6.5. For a fixed $x \in G$, define the normalized boundary value of $H_{\epsilon}(x)($.$) to be$

$$
\begin{aligned}
& {\left[\mathcal{B}_{M_{1}} H_{\epsilon}(x)\right](m)} \\
& \quad=\sum_{j} \gamma^{-1}\left(\bar{P} \cap M_{1}^{\#}, P \cap M_{1}^{\#}, \sigma, i \epsilon \rho_{M_{1}}\right) \Phi_{P \cap M_{1}^{\#}, 1}\left(\psi_{j}, \pi_{\sigma, i \epsilon \rho_{M_{1}}}\left(m^{-1}\right) F_{\epsilon}(x)(.)\right)
\end{aligned}
$$

with $\Phi_{P \cap M_{1}^{\#}, 1}(., .,$.$) as in \S 5(5.4)$.

By Proposition 5.2 we have

$$
\begin{gathered}
\mathcal{B}_{M_{1}}\left[H_{\epsilon}(x)\right](1) \\
=\gamma_{P \cap M_{1}^{\#}}^{-1} \sum_{i}\left\langle\mathcal{A}\left(\bar{P} \cap M_{1}^{\#}, P \cap M_{1}^{\#}, \sigma, i \epsilon \rho_{M_{1}}\right) F_{\epsilon}(x)(1), \phi_{i}\right\rangle_{V \sigma} \phi_{i} \\
\quad=\gamma_{P \cap M_{1}^{\#}}^{-1} \mathcal{A}\left(\bar{P} \cap M_{1}^{\#}, P \cap M_{1}^{\#}, \sigma, i \epsilon \rho_{M_{1}}\right) F_{\epsilon}(x)(1) .
\end{gathered}
$$

Definition 6.6. Define the boundary value of $\mathcal{S}_{\epsilon} f_{\epsilon}$ by means of

$$
\mathcal{B}\left[\mathcal{S}_{\epsilon} f_{\epsilon}\right](k)=\mathcal{B}_{M_{1}}\left[\lim _{a_{1} \rightarrow \infty, P_{1}} a_{1}^{\rho_{1}-\rho_{L}} \sigma\left(w_{0}\right) \mathcal{S}_{\epsilon} f_{\epsilon}\left(k a_{M_{1}} p a_{1}\right)\right] .
$$

By Proposition 5.2 and [W-2, page 191] we have

$$
\begin{aligned}
\mathcal{B}\left[\mathcal{S}_{\epsilon} f_{\epsilon}\right](k) & =\gamma_{P \cap M_{1}^{\#}}^{-1} \sigma\left(w_{0}\right) A_{P_{1}}\left(w_{0}, \pi_{\sigma, i \epsilon \rho_{M_{1}}},\left.\rho_{L}\right|_{\mathfrak{a}_{1}}\right) \\
& \circ \mathcal{A}\left(\bar{P} \cap M_{1}^{\#}, P \cap M_{1}^{\#}, \sigma, i \epsilon \rho_{M_{1}}\right) F_{\epsilon}(k)(1) \\
& =\sigma\left(w_{0}\right) \mathcal{A}_{P}\left(w_{0}, \sigma, \rho_{L}+i \epsilon \rho_{M_{1}}\right) f_{\epsilon}(k) .
\end{aligned}
$$

Definition 6.7. For $f \in \operatorname{ind}_{M \# A N}^{G}\left(\sigma, \rho_{L}, 1\right)$, we define the normalized boundary value of $\mathcal{S} f$ to be

$$
\mathcal{B}[\mathcal{S} f](k)=\lim _{\epsilon \rightarrow 0} \mathcal{B}\left[\mathcal{S}_{\epsilon} f_{\epsilon}\right](k)
$$

Thus, $\mathcal{B}[\mathcal{S} f](k)=\sigma\left(w_{0}\right) \mathcal{A}_{P}\left(w_{0}, \sigma, \rho_{L}\right) f(k)$.

Remark 6.8. The limit

$$
\begin{aligned}
& \lim _{\epsilon \rightarrow 0} \lim _{a_{M_{1}} \rightarrow \infty, P \cap M_{1}^{\#}} a_{M_{1}}^{\rho_{M_{1}}} \\
& \quad \times\left\{\gamma^{-1}\left(\bar{P} \cap M_{1}^{\#}, P \cap M_{1}^{\#}, \sigma, i \epsilon \rho_{M_{1}}\right) \lim _{a_{1} \rightarrow \infty, P_{1}} a_{1}^{\rho_{1}-\rho_{L}} \sigma\left(w_{0}\right) \mathcal{S}_{\epsilon} f_{\epsilon}\left(k a_{M_{1}} p a_{1}^{-1}\right)\right\}
\end{aligned}
$$

exists and can be given explicitly in terms of intertwining operators by using Proposition 5.3 in $\S 5$.

\section{Boundary VAlues of Szegö Integrals: THE DIFFERENT REAL RANK CASE}

In this section we drop the assumption real $\operatorname{rank} G=$ real rank $L$. In the spirit of $\S 6$, we consider

$$
\lim _{a \rightarrow \infty, P} a_{+}^{\rho_{L}-\rho_{G}} \mathcal{S} f\left(k a_{+}\right) .
$$

Since the cuspidal parabolic subgroup $P$ is not a minimal parabolic subgroup of $G$, in order to make sense of the limit $(7.1)$ we restrict our attention to $K$-finite elements in Image $\mathcal{S}$. 
The domain of the Szegö map consists of smooth vectors in

$$
\operatorname{ind}_{M \# A N}^{G}\left(\mathbb{D}(\psi, \lambda) \otimes \xi_{\lambda+2 \delta(\mathfrak{u} \cap p)}, \rho_{L}, 1\right)
$$

where $\mathbb{D}(\psi, \lambda) \otimes \xi_{\lambda+2 \delta(\mathfrak{u} \cap p)}$ is the $M^{\#}$-discrete series representation constructed in $\S 2$. As indicated in $\S 2$, we identify the representation $\mathbb{D}(\psi, \lambda) \otimes \xi$ with the representation of $M^{\#}$ acting on a subspace $\mathcal{J}_{M^{\#}}^{\chi}$ of the kernel of Schmid's differential operator $\mathcal{D}_{M \#}$.

Let $w_{0}=\pi s_{\mathbf{c} \alpha_{i}} \in W(G, A)$ and let $p \in K$ be a representative. The intertwining map

$$
\left[T\left(w_{0}\right) f\right](m)=P_{\chi}\left[\tau\left(p^{-1}\right) f(m)\right]
$$

where $P_{\chi}$ denotes the projection from $V_{\chi}$ to $\tilde{V}_{\chi}$, exhibits the representations of $M^{\#}$ acting on $C^{\infty}\left(M^{\#} /\left(K \cap M^{\#}\right), \tilde{V}_{\chi}\right)$ and $C^{\infty}\left(M^{\#} / p\left(K \cap M^{\#}\right) p^{-1}, \tilde{V}_{p\left(K \cap M^{\#}\right) p^{-1}}\right)$ as equivalent.

Remark 7.1. Let $f$ be a vector in $C^{\infty}\left(G,\left[(\mathbb{D}(\psi, \lambda) \otimes \xi], \rho_{L}, 1\right)\right.$. For each element $k \in K, f(k)($.$) is a smooth section of the vector bundle$

$$
M^{\#} \times_{M^{\#} \cap K} \tilde{V}_{\chi} \rightarrow M^{\#} /\left(M^{\#} \cap K\right) .
$$

Moreover, $f(k)(m)$ as a function of $m$ lies in a subspace $\mathcal{J}_{M^{\#}}^{\chi}$ of the kernel of the Schmid's operator $\mathcal{D}_{M}$.

If we assume that $f$ is a $K$-finite vector in $C^{\infty}\left(G,\left[(\mathbb{D}(\psi, \lambda) \otimes \chi], \rho_{L}, 1\right)\right.$, then $f(k)(.) \subset\left\{M^{\#} \cap K\right.$-finite vectors in $\left.\mathcal{J}_{M \#}^{\chi}\right\}$. (See, [W-1, 5.2].) It follows from work of Hotta-Parthasarathy [HP], Schmid [S], Knapp-Wallach [KW], that $M^{\#} \cap K$-finite vectors in $\mathcal{J}_{M^{\#}}^{\chi}$ are square integrable.

Let $l \in K$ and let $\psi_{1}, \ldots, \psi_{d}$ be a basis for span $\left\{R\left(M^{\#} \cap K\right) L\left(M^{\#} \cap K\right) f\right\}$. (Here, $R$ and $L$ denote right and left translation respectively.) Now, if $m_{1}, m_{2} \in$ $M^{\#} \cap K$ and $a^{*} \in A^{*}$, then

$$
f(l)\left(m_{1} a^{*} m_{2}\right)=\sum h_{j}(l)\left(m_{1}, m_{2}\right) \psi_{j}\left(a^{*}\right)
$$

with $h_{j}(l) \in C^{\infty}\left(\left(M^{\#} \cap K\right) \times\left(M^{\#} \cap K\right), \tilde{V}_{\chi}\right)$. Thus, there exists a constant $C$ such that

$$
\left|f(l)\left(m_{1} a^{*} m_{2}\right)\right| \leqq C \sum\left|\psi_{j}\left(a_{+}^{*}\right)\right|
$$

for all $l \in K, m_{1}, m_{2} \in\left(M^{\#} \cap K\right)$ and $a^{*} \in A_{+}^{*}$. Our previous observations imply that the smooth $\left(M^{\#} \cap K\right)$-finite functions $\psi_{j}$ are so that $X \psi_{j}$ are square integrable for all $X \in \mathcal{U}(\mathfrak{m})$. The asymptotic behavior of such functions is well understood. See for example [W-1, 5A 3.4]. We use the results in [W-1, 5A 3.4] and well known properties of the zonal spherical function $\Xi_{M}$ of $M$ to conclude that $f(l)(m)$ is bounded above by $\Xi_{M}(m)$.

Remark 7.2. Let $w_{0}=\pi s_{\mathbf{c} \alpha_{i}} \in W(G, A)$ and let $p \in K$ be a representative. Then

$$
\mathcal{S} f\left(k m a_{+}\right)=\tau(p) \mathcal{S} f\left(k p m_{1} a_{+}^{-1}\right), \quad \text { with } m_{1}=p^{-1} m p .
$$

The observations in Remarks 7.1 and 7.2 combined with an argument similar to the one used in Lemma 6.2 allow us to prove 
Proposition 7.3. If $\rho_{L}$ is in the open positive restricted Weyl chamber and $f$ is a $K$-finite vector of $C^{\infty}\left(G,[\mathbb{D}(\psi, \lambda) \otimes \chi], \rho_{L}, 1\right)$, then

$$
\lim _{a_{+} \rightarrow \infty, P} a^{\rho_{G}-\rho_{L}} P_{\chi} \mathcal{S} f\left(k m a_{+}\right)=\left[T\left(w_{0}\right) A_{P}\left(w_{0}, \rho_{L}, \mathbb{D}(\psi, \lambda) \otimes \xi\right) f(k)\right]\left(p^{-1} m p\right)
$$

for each $m \in M^{\#}$. The limit (6.9) converges pointwise and uniformly for $k \in K$.

As in $\S 6$ we can reinterpret the boundary value map as a (now infinitesimal) pointwise embedding of Image $\mathcal{S}$ in the principal series $\operatorname{ind}_{M A N}^{G}\left(\sigma,-\rho_{L}, 1\right)$ (provided $\rho_{L}$ is strictly dominant). Once again, the diagram of $(\mathfrak{g}, K)$-equivariant maps

$$
\operatorname{ind}_{M A N}^{G}\left(\sigma, \rho_{L}, 1\right) \stackrel{T\left(w_{0}\right) A_{P}\left(w_{0}, \sigma, \rho_{L}\right)}{\searrow} \operatorname{ind}_{M A N}^{G}\left(w_{0} \sigma,-\rho_{L}, 1\right)
$$

commutes. Let $(\operatorname{Im}(\mathcal{S}))_{K}$ and $\operatorname{Im}(\mathcal{B})_{K}$ denote the space of $K$-finite vectors in Image $\mathcal{S}$ and Image $\mathcal{B}$ respectively. Since the representations involved are finitely generated admissible modules, then the operator $\mathcal{B}$ extends to a continuous $G$ equivariant map from $\overline{(\operatorname{Im}(\mathcal{S}))_{K}}$ to the closure $\overline{(\operatorname{Im}(\mathcal{B}))_{K}}$.

The analysis in Proposition 7.3 runs into difficulties when $\rho_{L}$ is dominant singular. As in $\S 6$, we first associate to each $K$-finite function

$$
f \in \operatorname{ind}_{M \# A N}^{G}\left(\mathbb{D}(\psi, \lambda) \otimes \xi, \rho_{L}, 1\right)
$$

a $K$-finite function

$$
F \in \operatorname{ind}_{M_{1}^{\#} A_{1} N_{1}}^{G}\left(\operatorname{ind}_{P \cap M_{1}^{\#}}^{M_{1}^{\#}}(\mathbb{D}(\psi, \lambda) \otimes \xi, 0,1),\left.\rho_{L}\right|_{\mathfrak{a}_{1}}, 1\right)
$$

so that

$$
\mathcal{S} f(x)=\mathcal{S}_{1} F(x)=\int_{K} \tau(k)[F(x k)(1)(1)] d k .
$$

Next, for each $x \in G, m \in M^{\#}$, we set

$$
\mathcal{S} f(x)(m)=\int_{K} \tau(k)[f(x k)(m)] d k .
$$

$\mathcal{S} f().($.$) is an "extension" of the Szegö map in that \mathcal{S} f(x)(1)=\mathcal{S} f(x)$. For each $K \cap M^{\#}$-finite vector $\Phi$ in $\mathcal{J}_{M^{\#}}^{\chi}$, we consider the inner product

$$
\langle\mathcal{S} f(x)(.), \Phi(.)\rangle_{L^{2}\left(M^{\#}\right)} \text {. }
$$

Remark 7.4. If $\Psi$ is the function defined by means of

$$
\Psi(k)\left(m_{1}\right)(m)=\int_{M^{\#} \cap K} \tau_{\chi}^{1}\left(m_{1} l\right)^{-1} \tau_{\chi}(k)^{-1} \Phi\left(l^{-1} m\right) d l
$$

for $\left(k, m_{1}, m\right) \in\left(K \times\left(K \cap M_{1}^{\#}\right) \times M^{\#}\right)$, then it is easy to check that $\Psi$ gives rise to a $K$-finite vector in $\operatorname{ind}_{M_{1}^{\#} A_{1} N_{1}}^{G}\left(\left.\operatorname{ind}_{P \cap M_{1}^{\#}}^{M_{1}^{\#}}(\mathbb{D}(\psi, \lambda) \otimes \xi, 0,1) \rho_{L}\right|_{\mathfrak{a}_{1}}, 1\right)$. Moreover,

$$
\begin{aligned}
\langle\mathcal{S} f(x)(.), \Phi(.)\rangle_{L^{2}\left(M^{\#}\right)} & =\int_{K} \int_{K \cap M_{1}^{\#}}\left\langle F(x k)\left(m_{1}\right)(.), \Psi(k)\left(m_{1}\right)(.)\right\rangle_{L^{2}\left(M^{\#}\right)} d m_{1} d k \\
& =\left\langle U\left(P_{1}, \pi_{\sigma, 0},\left.\rho_{L}\right|_{\mathfrak{a}_{1}}\right)\left(x^{-1}\right) F, \Psi\right\rangle .
\end{aligned}
$$


Proposition 7.5. We maintain the above assumptions and notation. Let $f$ be a $K$-finite function in $\operatorname{ind}_{M_{A N}^{\#}}^{G}\left(\mathbb{D}(\psi, \lambda) \otimes \xi, \rho_{L}, 1\right)$ and let $\Phi$ be a $K \cap M^{\#}$-finite vector in $\mathcal{J}_{M^{\#}}^{\chi}$. Fix $a_{M_{1}} \in A_{M_{1}}^{+}$and let $a_{1} \in A_{1}^{+}$. Then,

$$
\begin{aligned}
\lim _{a_{1} \rightarrow \infty, P_{1}} a_{1}^{\rho_{1}-\rho_{l}}\left\langle\mathcal{S} f\left(k a_{M_{1}} a_{1}\right)(.), \Phi(.)\right\rangle_{L^{2}\left(M^{\#}\right)} \\
=\left\langle A\left(\bar{P}_{1}, P_{1}, \pi_{\sigma, 0},\left.\rho_{L}\right|_{\mathfrak{a}_{1}}\right) F\left(k a_{M_{1}} p\right), \Psi(p)\right\rangle .
\end{aligned}
$$

Proof. This is immediate from Remark 7.4 and a result of Langlands. (See for example [K-2, pg.199] or [W-1, 5.3.4].)

Proposition 7.6. For each $\bar{n}_{1} \in \bar{N}_{1}$, let $\Phi_{P \cap M_{1}^{\#}}\left(a_{M_{1}}, \Phi, F\left(k p \bar{n}_{1}\right)().\right)$ be the leading term in the asymptotic expansion of $\left\langle\pi_{\sigma, 0} F\left(k p \bar{n}_{1}\right)(),. \Phi().\right\rangle$. Then

$$
\begin{aligned}
\lim _{a_{M_{1}} \rightarrow \infty, P \cap M_{1}^{\#}} \| \lim _{a_{1} \rightarrow \infty, P_{1}} a_{M_{1}}^{\rho_{M_{1}}} a_{1}^{\rho_{1}-\rho_{L}}\left\langle\mathcal{S} f\left(k a_{M_{1}} a_{1}\right), \Phi\right\rangle \\
\quad-\int_{\bar{N}_{1}} \Phi_{P \cap M_{1}^{\#}}\left(a_{M_{1}}, \tau\left(p^{-1}\right) \Phi, F\left(k p \bar{n}_{1}\right)(.) d \bar{n}_{1} \|=0 .\right.
\end{aligned}
$$

Proof. Similar to Proposition 6.4

Let $\epsilon>0$, and consider the principal series representation

$$
\operatorname{ind}_{M{ }^{\#} A N}^{G}\left(\mathbb{D}(\Psi, \lambda) \otimes \chi, \rho_{L}+\epsilon \rho_{M_{1}}, 1\right) .
$$

If $f_{\epsilon} \in \operatorname{ind}_{M A_{A N}}^{G}\left(\mathbb{D}(\Psi, \lambda) \otimes \chi, \rho_{L}+\epsilon \rho_{M_{1}}, 1\right)$, then define

$$
F_{\epsilon} \in \operatorname{ind}_{M_{1}^{\#} A_{1} N_{1}}^{G}\left(\operatorname{ind}_{M_{1}^{\#} \cap P}^{M_{1}^{\#}}\left(\mathbb{D}(\Psi, \lambda) \otimes \chi, \epsilon \rho_{M_{1}}, 1\right),\left.\rho_{L}\right|_{\mathfrak{a}_{1}}, 1\right)
$$

by $F_{\epsilon}(x)(m)=f_{\epsilon}(x m)$.

For each $K \cap M^{\#}$-finite vector $\Phi$ in $\mathcal{J}_{M \#}^{\chi}$, we have

$$
\begin{aligned}
& \left\langle\mathcal{S}_{\epsilon} f_{\epsilon}(x)(.), \Phi(.)\right\rangle_{L^{2}\left(M^{\#}\right)} \\
& \quad=\left\langle U\left(P_{1}, \pi_{\mathbb{D}(\Psi, \lambda) \otimes \chi, i \epsilon \rho_{M_{1}}},\left.\rho_{L}\right|_{\mathfrak{a}_{1}}\right)\left(x^{-1}\right) F_{\epsilon}(.), \Psi(.)\right\rangle_{L^{2}\left(M^{\#}\right)} .
\end{aligned}
$$

Here $\Phi$ and $\Psi$ are as in Remark 7.4.

In what follows we assume that the $f_{\epsilon}$ transform according to a representation of $K$ (on the left).

Definition 7.7. For a fixed $x \in G$, define the normalized boundary value of $F_{\epsilon}(x)($.$) by$

$$
\begin{aligned}
\mathcal{B}_{M_{1}} & F_{\epsilon}(x)\left(m_{1}\right)(.) \\
= & \sum_{j} \Phi_{P \cap M_{1}^{\#}}\left(\lambda_{\omega_{j}}, \pi_{\mathbb{D}(. .,) \otimes \chi, i \epsilon \rho_{M_{1}}}\left(m_{1}^{-1}\right) F_{\epsilon}(x)(1)\right) \\
& \quad \times \gamma^{-1}\left(\bar{P} \cap M_{1}^{\#}, P \cap M_{1}^{\#}, \mathbb{D}(\Psi, \lambda) \otimes \chi, i \epsilon \rho_{M_{1}^{\#}}\right) \omega_{j}(1)(.)
\end{aligned}
$$

with $\omega_{j}$ ranging over an orthonormal basis of the subspace of

$$
\operatorname{ind}_{M_{1}^{\#} \cap P}^{M_{1}^{\#}}\left(\mathbb{D}(\Psi, \lambda) \otimes \chi, i \epsilon \rho_{M_{1}}, 1\right)
$$

containing all images in $\operatorname{ind}_{M_{1}^{\#} \cap P}^{M_{1}^{\#}}\left(\mathbb{D}(\Psi, \lambda) \otimes \chi, i \epsilon \rho_{M_{1}}, 1\right)$ of members of the induced space transforming according to the given $K$-type. 
Then, by Proposition 5.2 we have

$$
\begin{aligned}
& \mathcal{B}_{M_{1}}\left(F_{\epsilon}(x)\right)(1)(m)=\gamma_{P \cap M_{1}^{\#}}^{-1} \\
& \times \sum_{j}\left\langle\mathcal{A}\left(\bar{P} \cap M_{1}, P \cap M_{1}, \mathbb{D}(\Psi, \lambda) \otimes \chi, i \epsilon \rho_{M_{1}}\right) F_{\epsilon}(x)(1), \omega_{j}(1)\right\rangle_{L^{2}\left(M^{\#}\right)} \omega_{j}(1)(m) .
\end{aligned}
$$

Definition 7.8. Define the boundary value of $\mathcal{S}_{\epsilon} f_{\epsilon}$ by means of

$$
\mathcal{B}\left(\mathcal{S}_{\epsilon} f_{\epsilon}\right)(1)=\mathcal{B}_{M_{1}}\left[\sum_{j} \lim _{\left(a_{1} \rightarrow \infty, P\right)}\left\langle T\left(\omega_{o}\right) \mathcal{S}_{\epsilon}\left(f_{\epsilon}\right)\left(k a_{M_{1}} p a_{1}\right), \omega_{j}\right\rangle_{L^{2}\left(M^{\#}\right)} \omega_{j}(1)\right] .
$$

Thus by Proposition 7.6 and [W-2, page 191] the above is

$$
\begin{aligned}
& \gamma_{P \cap M_{1}^{\#}}^{-1} \times \sum_{j}\left\langle\pi_{\mathbb{D} \otimes \chi, i \epsilon \rho_{M_{1}}}(p) A_{P}\left(\omega_{o},\left.\pi_{\mathbb{D} \otimes \chi, i \epsilon \rho_{M_{1}}} \rho_{L}\right|_{\mathfrak{a}_{1}}\right)\right. \\
& \left.\circ \mathcal{A}\left(\bar{P} \cap M_{1}^{\#}, P \cap M_{1}^{\#}, \mathbb{D}(\Psi, \lambda) \otimes \chi, i \epsilon \rho_{M_{1}}\right) f_{\epsilon}(k p)(.), \omega_{j}(1)(.)\right\rangle_{L^{2}\left(M_{1}^{\#}\right)} \omega_{j}(1)(.) \\
& =\sum_{j}\left\langle\mathbb{D} \otimes \chi(p) \mathcal{A}_{P}\left(\omega_{o}, \mathbb{D}(\Psi, \lambda) \otimes \chi, \rho_{L}+i \epsilon \rho_{M_{1}}\right) f_{\epsilon}(k)(.), \omega_{j}(1)(.)\right\rangle_{L^{2}\left(M^{\#}\right)} \omega_{j}(1)(.) .
\end{aligned}
$$

Definition 7.9. If $f \in \operatorname{ind}_{M \# A N}^{G}\left(\mathbb{D}(\Psi, \lambda) \otimes \chi, \rho_{L}, 1\right)$ transforms according to a representation of $K$ on the left, then define the normalized boundary value of $\mathcal{S} f$ to be

$$
\mathcal{B}[\mathcal{S} f](k)=\lim _{\epsilon \rightarrow 0} \mathcal{B}\left[\mathcal{S}_{\epsilon} f_{\epsilon}\right](k)
$$

Thus,

$$
\begin{aligned}
& \mathcal{B}[\mathcal{S} f](k)(m) \\
& =\sum_{j}\left\langle\mathbb{D}(\Psi, \lambda) \otimes \chi(p) \mathcal{A}_{P}\left(\omega_{o},(\Psi, \lambda) \otimes \chi, \rho_{L}\right) f(k)(.), \omega_{j}(1)(.)\right\rangle_{L^{2}\left(M^{\#}\right)} \omega_{j}(1)(m) .
\end{aligned}
$$

\section{REFERENCES}

[Bar] V. Bargman, Irreducible unitary representations of the Lorentz group, Ann. of Math. 48 (1947), 586-640. MR 9:133a

[Bl-1] B. Blank, Embedding limits of discrete series of semisimple Lie groups, Canadian Math. Conf. Proc. 1 (1981), 55-64. MR 83h:22001

[Bl-2] B. Blank, Knapp-Wallach Szegö integrals and the P-induced continuous representation: The parabolic rank one case, J.Func.Anal. 60 (1985), 127-145. MR 86i:22032

[Bl-3] B. Blank, Boundary behavior of limits of discrete series representations of real rank one groups, Pacific Jour. of Math. 122 (1986), 299-318. MR 87e:22027

[Bl-4] B. Blank, Knapp-Wallach Szegö integrals II, The higher parabolic rank case, Trans. A.M.S 300 (1987), 49-59. MR 88f:22041

[BKZ] L. Barchini, A.W. Knapp and R. Zierau, InterTwining operators into Dolbeault cohomology, Jour. Func. Anal. 107 (1992), 302- 341. MR 93e:22026

[B-1] L. Barchini, Szegö mappings, harmonic forms and Dolbeault cohomology, Jour. Func. Anal. 118 (1993), 351-406. MR 94k:22033

[B-2] L. Barchini, Szegö kernels associated with Zuckerman modules, J. Func. Anal. 131 (1995), 145-181. MR 96i:22033

[BW] A. Borel and N. Wallach, Continuous cohomology, discrete subgroups, and representations of reductive groups, Annals of Math. Studies, no. 94, Princeton Univ. Press, Princeton, NJ, 1980. MR 83c:22018

[C] W. Casselman, The differential equations satisfied by matrix coefficients, manuscript (1975). 
[GKST] J.E. Gilbert, A. Kunze, R.J. Stanton, and P. Thomas, Higher gradients and representations of Lie groups, Conference on Harmonic Analysis in honor of Antoni Zygmund, vol. II, Wadsworth, Belmont, CA, 1983, pp. 416-436. MR 85k:22032

[HC-1] Harish-Chandra, Harmonic Analysis on Real Reductive Groups I, the theory of the constant term, J.Func.Anal. 19 (1975), 104-204. MR 53:3201

[HC-2] Harish-Chandra, Harmonic Analysis on Real Reductive Groups II, Invent. Math. 36 (1976), 1-55. MR 55:12874

[HP] R. Hotta and R. Parthasarathy, Multiplicity formulae for discrete series, Invent. Math. 26 (1974), 133-178. MR 50:539

[KKMOOT] M. Kashiwara, A. Kowata, K. Minemura, K. Okamoto, T. Oshima and M. Tanaka, Eigenfunctions of invariant differential operators on a symmetric space, Ann. of Math. 107 (1978), 1-39. MR 81f:43013

[Ka] T. Kawazoe, On a global realization of a discrete series for $S U(n, 1)$ as applications of Szegö operators and limits of discrete series, Tokyo J.Math. 12 (1989). MR 91b:22020

[K-O] A.W. Knapp and K. Okamoto, Limits of holomorphic discrete series, J. Func.Anal. 9 (1972), 375-409. MR 45:8774

[K-1] A.W. Knapp, A Szegö kernel for discrete series, Proceedings International Congress of Mathematicians. Canadian Mathematical Congress. 2 (1975), 99-104. MR $\mathbf{5 5 : 1 0 6 0 6}$

[KW] A.W. Knapp and N. Wallach, Szegö kernels associated to discrete series, Invent. Math. 34, 62 (1976, 1980), 163-200, 341-346. MR 54:7704; MR 82i:22016

[K-2] A.W. Knapp, Commutativity of Intertwining Operators for Semisimple Lie Groups, Compositio Math. 46 (1982), 33-84. MR 83i:22022

[KS] A.W. Knapp and E. Stein, Intertwining operators for semisimple groups II, Invent. Math. 60 (1980), 9-84. MR 82a:22018

[KZ] A.W. Knapp and G. Zuckerman, Classification of irreducible tempered representations of semisimple groups, Ann. of Math. 116 (1982), 389-455. MR 84h:22034a

[K-3] A.W. Knapp, Representation Theory of Semisimple Groups : An overview based on examples, Princeton Univ. Press, Princeton, NJ, 1986. MR 87j:22022

[L] R. Langlands, On the classification of irreducible representations of real algebraic groups, Mimeographed notes, Inst. Adv. Stud., Princeton, NJ, 1973; reprinted in Representation theory and harmonic analysis on semisimple Lie groups (P.J. Sally, Jr., and D. A. Vogan, eds.), Math. Surveys and Monographs, vol. 31, Amer. Math. Soc., Providence, RI, 1989, pp. 101-170. MR 91e:22017

[Ma] H. Matumoto, Cohomological Hardy spaces for $S U(2,2)$, Adv. Studies in Pure Math. 14 (1988), 469-497. MR 91c:22032

[Mi] H. Midorikawa, On certain irreducible representations of real rank one classical groups, J.Fac.of Science, The Univ. Tokyo, Sec IA. 27, no. 3 (1974), 435-459. MR 51:809

[M] D. Milicic, Asymptotic behavior of matrix coefficients of discrete series, Duke Math.J. 44 (1977), 59-88. MR 55:3171

[S] W. Schmid, Homogeneous complex manifolds and representations of semisimple Lie groups, Ph.D. thesis, Univ. of California, Berkeley, CA, 1967; reprinted in Representation theory and harmonic analysis on semisimple Lie groups (P. J. Sally, Jr., and D. A. Vogan, Jr., eds.), Math. Surveys and Monographs, vol. 31, Amer. Math. Soc., Providence, RI, 1989, pp. 223-286. MR 90i:22025

[Ta] R.Takashashi, Sur les fonctions spheriques et la formulae de Plancherel dans le groupe hyperbolique, Jap. J.Math. 31 (1961), 55-90. MR 27:2809

[VZ] D. Vogan and G.J. Zuckerman, Unitary representations with continuous cohomology, Compositio Math. 53 (1984), 51-90. MR 86k:22040

[W-1] N. Wallach, Asymptotic expansion of generalized matrix entries of representations of real reductive groups, Lie Group Representations, I (College Park, MD, 1982/83), Lecture Notes in Math., vol. 1024, Springer-Verlag, Berlin, 1983, pp. 287-369. MR 85g:22029

[W-2] N. Wallach, Real Reductive Groups I, Academic Press, 1988. MR 89i:22029 
[W-3] N. Wallach, Real Reductive Groups II, Academic Press, 1992. MR 93m:22018

[Wo] H-W. Wong, Dolbeault cohomologies and Zuckerman modules associated with finite rank representations, Ph.D. thesis, Harvard Univ., Cambridge, MA, 1991. See also J. Funct. Anal. 129 (1995), 428-454. MR 96c:22024

Department of Mathematics, Temple University, Philadelphia, Pennsylvania 19122

Current address: Department of Mathematics, Oklahoma State University, Stillwater, Oklahoma 74078

E-mail address: leticiz@math.okstate.edu 